\title{
Spectral sets of periodic matrices related to the strong moment problem
}

\author{
Ionela Moale and Peter Yuditskii ${ }^{1}$
}

\begin{abstract}
The main result of this work is a parametric description of the spectral sets of a class of periodic 5-diagonal matrices, related to the strong moment problem. This class is a self-adjoint twin of the class of CMV matrices. Both are hidden in the simplest possible class of regular 5-diagonal matrices, this fact we also show here.
\end{abstract}

Mathematics Subject Classification (2010). 30E05, 30F15, 47B39, 46E22.

Keywords. Strong moment problem, periodic CMV matrices, Hardy spaces on Riemann surfaces, conformal mappings, comb domains, reproducing kernels.

\section{Contents}

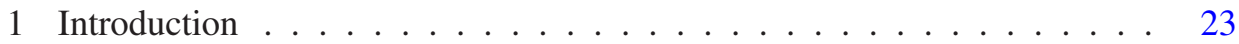

2 Spectral surfaces with the maximal number of boundary ovals $\ldots . .33$

3 Proof of the Main Theorem . . . . . . . . . . . . . . . . . . . 40

4 Functional model for periodic SMP matrices . . . . . . . . . . . 45

References . . . . . . . . . . . . . . . . . . . . . . . 49

\section{Introduction}

1.1. Historical remarks; towards the setting of the problem. Akhieser and Levin introduced special conformal mappings on so-called comb-like domains, see [1]. Marchenko and Ostrovskii [17] used successfully these conformal mappings in the spectral theory of periodic Sturm-Liouville operators, in particular they gave a parametric description of the spectral sets of such operators. Later this idea was adopted by Perkolab in [28] to periodic Jacobi matrices. On the other hand, these conformal

\footnotetext{
${ }^{1}$ This work was supported by Austrian Science Fund FWF, project no: P22025-N18.
} 
mappings are strongly connected to Chebyshev-type extremal problems; this connection was explicitly noted by Peherstorfer in [20], [21], and [22]; see also [31]. In connection to these extremal problems, see also [3] and [4].

Spectral properties of multi-diagonal and block-Jacobi matrices, as well as their continuous analogues, like Schrödinger operators with periodic matrix potentials were studied intensively; for essential results in this direction see e.g. [7], [8], [12], and [16]. Let's note that a new very interesting application of block-Jacobi matrices was found by Damanik, Killip and Simon [9].

Our problem is related to the general objective of finding a parametric description of spectral surfaces corresponding to multi-diagonal periodic matrices. We will try to clarify the meaning of the words "parametric description." Following Marchenko, we would like to have a description by free independent parameters subject to at most trivial restrictions. Generally, these spectral surfaces were described in the classical paper [18] of Mumford and van Moerbeke, however this description hardly fits to the above mentioned criterion of Marchenko. Let us discuss both descriptions in the simplest possible case of periodic Jacobi matrices.

Consider a doubly infinite, periodic Jacobi matrix

$$
J=\left[\begin{array}{ccccc}
\ddots & & & & \\
\ddots & p_{-1} & & & \\
\ddots & q_{-1} & p_{0} & & \\
& p_{0} & q_{0} & p_{1} & \\
& & p_{1} & q_{1} & \ddots \\
& & & p_{2} & \ddots \\
& & & & \ddots
\end{array}\right],
$$

where $p_{k+N}=p_{k}, q_{k+N}=q_{k}, p_{k}>0$, and $q_{k} \in \mathbb{R}$. This matrix defines a bounded self-adjoint operator on $\ell^{2}$. It is well-known that the spectrum consists of the union of non-degenerated intervals $E=\left[b_{0}, a_{0}\right] \backslash \bigcup_{j \geq 1}\left(a_{j}, b_{j}\right)$, see e.g. [28], but it is far from being an arbitrary system of intervals. So, the point is to give a parametric description of those sets which can be the spectrum of periodic Jacobi matrices. First we recall such a description according to the approach of Marchenko and Ostrovskii. For a system of nonnegative parameters $\left\{h_{k}\right\}_{k=1}^{n-1}$, let $D=D\left(h_{1}, \ldots, h_{n-1}\right)$ be the region obtained from the half-strip

$$
\{w:-\pi n<\operatorname{Re} w<0, \operatorname{Im} w>0\}
$$

by removing vertical intervals

$$
\left\{w: \operatorname{Re} w=-\pi k, 0<\operatorname{Im} w \leq h_{k}\right\}, \quad k=1, \ldots, n-1 .
$$


Such a domain is called a comb; furthermore, we call the interval $[-\pi n, 0]$ the base of the comb, the half-axes

$$
\{w: \operatorname{Re} w=0, \operatorname{Im} w \geq 0\}
$$

and

$$
\{w: \operatorname{Re} w=-\pi n, \operatorname{Im} w \geq 0\}
$$

form its right and left sides, and the slits (1.1) are called the teeth of the comb.

Let $\theta$ be the conformal map of the upper half-plane $\mathbb{t}$ to $D$ normalized by the conditions $\theta\left(a_{0}\right)=0, \theta\left(b_{0}\right)=-\pi n, \theta(\infty)=\infty$, the so-called comb-function. Denote by $E\left(h_{1}, \ldots, h_{n-1}\right)$ the full preimage of the comb's base, i.e.:

$$
E\left(h_{1}, \ldots, h_{n-1}\right) \stackrel{\text { def }}{=} \theta^{-1}([-\pi n, 0]) .
$$

A system of intervals is the spectrum of a periodic Jacobi matrix if and only if $E=E\left(h_{1}, \ldots, h_{n-1}\right)$ for a certain system of parameters $\left\{h_{k}\right\}_{k=1}^{n-1}$. Let us point out that for fixed $a_{0}$ and $b_{0}$, for the spectral set we have $n-1$ free parameters $\left(h_{j} \geq 0, j=1, \ldots, n-1\right)$, meanwhile a general system of intervals is described by $2(n-1)$ parameters $\left(a_{j}, b_{j}, j=1, \ldots, n-1\right)$.

Next, we give the description of such sets in the language of the theorem of Mumford and van Moerbeke. To this end, we need to consider the two-sheeted Riemann surface

$$
\mathcal{R}=\left\{(w, z): w^{2}=\prod_{j \geq 0}\left(z-a_{j}\right)\left(z-b_{j}\right)\right\} .
$$

This surface should be compactified by adding two infinite points $\infty_{ \pm}$(on the upper and lower sheet). The corresponding system of intervals $E$ is the spectrum of a periodic Jacobi matrix if and only if the divisor $n \infty_{+}-n \infty_{-}$is a divisor of a function on $\mathcal{R}$. We have thus also a description of spectral sets, but from our point of view not by means of free independent parameters.

On the same level of explicitness, one can describe spectral sets of periodic CMV matrices (Chapter 11 in [30]). Notice that the spectral theory of CMV matrices, including the one of periodic matrices, and the related theory of orthogonal polynomials on the unit circle, see [2], [23], [24], and [25], became hot research topics in the last few years, see e.g. [5], [13], [14], and [15] and the books [29] and [30]. Going back to the spectrum of periodic CMV matrices, for a system of nonnegative parameters $\left\{h_{k}\right\}_{k=0}^{n-1}$ we define a periodic comb in the following way. Let

$$
D_{\mathrm{CMV}}=D_{\mathrm{CMV}}\left(h_{0}, \ldots, h_{n-1}\right)
$$

be the region obtained from the upper half-plane by removing vertical intervals (teeth)

$$
\left\{w: \operatorname{Re} w=2 \pi(k+n s), 0<\operatorname{Im} w \leq h_{k}\right\}, \quad k=0, \ldots, n-1, s \in \mathbb{Z} .
$$


Let $\theta_{\mathrm{CMV}}$ be the conformal map of the upper half-plane $\mathbb{U}$ to $D_{\mathrm{CMV}}$ normalized by the following asymptotics at infinity

$$
\theta_{\mathrm{CMV}}(z)=n z+i q+o(1), \quad z=x+i y, y \rightarrow \infty,
$$

where $q$ is positive. In this case the spectral set $E_{\mathrm{CMV}} \subset \mathbb{T}$ also corresponds to the base of the comb, given by

$$
E_{\mathrm{CMV}}\left(h_{0}, \ldots, h_{n-1}\right) \stackrel{\text { def }}{=}\left\{e^{i z}: z \in \theta_{\mathrm{CMV}}^{-1}(\mathbb{R})\right\} .
$$

Note that CMV matrices are already 5-diagonal, but of very specific structure.

The main result of this work is a parametric description of the spectral sets of another special class of periodic 5-diagonal matrices, which is a self-adjoint twin of the CMV class. They are related to the strong moment problem, see e.g. [19] and [6], and we take a risk to introduce an abbreviation and call them SMP matrices.

We would like to stress that certain general facts from their spectral theory can be immediately reduced to the spectral theory of Jacobi matrices, in the same way like corresponding properties of CMV matrices can be reduced to those of Jacobi matrices and vice versa. Indeed, let $J$ be a periodic Jacobi matrix. We define

$$
\tilde{\mathfrak{A}} \stackrel{\text { def }}{=} \frac{J-z_{0}}{J-\bar{z}_{0}}, z_{0} \in \mathbb{W} .
$$

Then $\widetilde{\mathfrak{A}}$ is an unitary operator, which is unitarily equivalent to a finite-band almost periodic CMV matrix $\mathfrak{A}$, see e.g. [26]. Or vice versa, let $\mathfrak{A}$ be a periodic CMV matrix and assume that $t_{0} \in \mathbb{T}$ does not belong to its spectrum. Then we define

$$
\widetilde{J} \stackrel{\text { def }}{=} i \frac{\mathfrak{A}+t_{0}}{\mathfrak{A}-t_{0}} .
$$

This matrix is unitarily equivalent to a finite-band almost periodic Jacobi matrix $J$. For this reason for instance, the spectrum of $J$ (a union of intervals) one can easily get by fractional linear transformation from the spectrum of $\mathfrak{A}$ (a union of arcs), and of course the spectral multiplicity (equal two) is the same for both matrices. However, we point out that a periodic matrix corresponds to an almost periodic one, and therefore there is no way to describe the spectral sets of such periodic Jacobi matrices by means of spectral sets of such CMV matrices, and vice versa. Both problems should be treated separately.

The same is related to SMP matrices. Certain general problems of their spectral theory, like multiplicity of the spectrum, can be easily reduced to the spectral theory of Jacobi matrices. Nevertheless, a description of the spectral set of periodic SMP matrices can be reduced to neither periodic CMV nor periodic Jacobi matrices. Our main result is such a description on the same level of explicitness like (1.2) and (1.4). 
1.2. SMP matrices, main problem. One-sided Jacobi matrices deal with orthogonal polynomials on the real axis. CMV matrices deal with orthogonalization of the following family of rational functions [29], p. 262,

$$
1, \lambda, \frac{1}{\lambda}, \lambda^{2}, \frac{1}{\lambda^{2}}, \ldots,
$$

with respect to a measure on the unit circle. One sided SMP matrices are related to orthogonalization of the same family (1.5) but with respect to a measure on the real axis. Note that even more general orthogonal systems of rational functions are still under investigations, see e.g. the most recent [10]. Further, a Jacobi matrix is not necessary associated to a measure with a compact support, but we are restricted to this kind of measures if we are interested in the Jacobi matrices generating bounded operators. Similarly, in the SMP matrix case we consider measures $\chi$ supported on a compact set without a vicinity of the origin. That is, not only operator multiplication by the independent variable $\lambda$ is bounded, but its inverse, the multiplication by $1 / \lambda$, is also bounded in $L_{\chi}^{2}$.

Let

$$
\varphi_{2 n-1}=\lambda^{n}+\cdots, \quad \varphi_{2 n}=\frac{1}{\lambda^{n}}+\cdots
$$

be the SMP-orthogonal system. Then for even vectors of the corresponding orthonormal basis we have the three term recurrence relations

$$
\lambda \frac{\varphi_{2 n}}{\left\|\varphi_{2 n}\right\|}=p_{2 n} \frac{\varphi_{2 n-1}}{\left\|\varphi_{2 n-1}\right\|}+q_{2 n} \frac{\varphi_{2 n}}{\left\|\varphi_{2 n}\right\|}+p_{2 n+1} \frac{\varphi_{2 n+1}}{\left\|\varphi_{2 n+1}\right\|}, \quad p_{0} \stackrel{\text { def }}{=} 0 .
$$

In the same time for odd vectors the three terms relations are generated by the multiplication by $1 / \lambda$ :

$$
\frac{1}{\lambda} \frac{\varphi_{2 n-1}}{\left\|\varphi_{2 n-1}\right\|}=\pi_{2 n-1} \frac{\varphi_{2 n-2}}{\left\|\varphi_{2 n-2}\right\|}+\sigma_{2 n-1} \frac{\varphi_{2 n-1}}{\left\|\varphi_{2 n-1}\right\|}+\pi_{2 n} \frac{\varphi_{2 n}}{\left\|\varphi_{2 n}\right\|} .
$$

The SMP matrix $A$ acting in $\ell_{+}^{2}$ is the matrix of the multiplication by $\lambda$ in $L_{\chi}^{2}$ with respect to the orthonormal basis $\frac{\varphi_{2 n}}{\left\|\varphi_{2 n}\right\|}$. Then, according to the above definition

$$
A e_{2 n}=A_{2} e_{2 n}=p_{2 n} e_{2 n-1}+q_{2 n} e_{2 n}+p_{2 n+1} e_{2 n+1}
$$

and

$$
A^{-1} e_{2 n-1}=A_{1} e_{2 n-1}=\pi_{2 n-1} e_{2 n-2}+\sigma_{2 n-1} e_{2 n-1}+\pi_{2 n} e_{2 n}
$$

where

$$
A_{1}=\left[\begin{array}{cccc}
1 & \pi_{1} & & \\
0 & \sigma_{1} & 0 & \\
& \pi_{2} & 1 & \ddots \\
& & 0 & \ddots \\
& & & \ddots
\end{array}\right], \quad A_{2}=\left[\begin{array}{cccc}
q_{0} & 0 & & \\
p_{1} & 1 & p_{2} & \\
& 0 & q_{2} & \ddots \\
& & p_{3} & \ddots \\
& & & \ddots
\end{array}\right],
$$


and $\left\{e_{n}\right\}_{n \geq 0}$ is the standard basis in $\ell_{+}^{2}$. In a generic position $A_{1}$ and $A_{2}$ are invertible, equivalently $\sigma_{2 n-1} \neq 0$ and $q_{2 n} \neq 0$ for all $n$, and we obtain the five-diagonal matrix in the form $A=A_{2} A_{1}^{-1}$. Due to the symmetry $A^{*}=A$ we have

$$
\frac{p_{2 n-1}}{q_{2 n-2}}=-\frac{\pi_{2 n-1}}{\sigma_{2 n-1}} \quad \text { and } \quad \frac{p_{2 n}}{q_{2 n}}=-\frac{\pi_{2 n}}{\sigma_{2 n-1}} .
$$

That is, in a generic case the one sided SMP matrix $A$, similarly to a Jacobi matrix, is given by two sequences $\left\{p_{2 n}, \pi_{2 n+1}\right\}_{n \geq 0}$ and $\left\{q_{2 n}, \sigma_{2 n+1}\right\}_{n \geq 0}$, since $p_{2 n+1}$ and $\pi_{2 n}$ can be found from (1.9).

Remark 1.1. A degeneration is possible: for instance, if the measure $\chi$ is symmetric, then $\varphi_{0}=1, \varphi_{1}=\lambda$, and therefore, $\pi_{2}=\sigma_{1}=0$. Of course, the operator $A$ and its inverse $A^{-1}$, given by 5-diagonal matrices, still have perfect sense, they just can not be written in the form $A_{2} A_{1}^{-1}$ and/or $A_{1} A_{2}^{-1}$ respectively. On the other hand, a degeneration is not possible if we assume that the operator $A$ is positive. But our main problem becomes especially interesting if the spectral set contains positive and negative components. It is very essential: we do not assume that $A>0$ !

Remark 1.2. The system $\varphi_{n}$ is just another orthogonal basis in $L_{\chi}^{2}$ comparably to the basis of orthogonal polynomials $P_{n}$. Therefore $A$ is unitarily equivalent to the Jacobi matrix $J$, which corresponds to the same operator of multiplication by the independent variable in $L_{\chi}^{2}$ in the basis $\left\{P_{n}\right\}_{n \geq 0}$.

As it is well known, two sided matrices are much more relevant to the periodic case. We postpone the formal definition of two sided matrices of the SMP class till Section 2, see Definition 2.5; but in a generic case, a SMP matrix is still defined as a ratio of two 3-diagonal two sided matrices $A=A_{2} A_{1}^{-1}$, where

$$
A_{1}=\left[\begin{array}{cccccc}
\ddots & & & & & \\
\ddots & \bar{\pi}_{-1} & & & & \\
\ddots & \sigma_{-1} & 0 & & & \\
& \pi_{0} & 1 & \bar{\pi}_{1} & & \\
& & 0 & \sigma_{1} & 0 & \\
& & & \pi_{2} & 1 & \ddots \\
& & & & 0 & \ddots \\
& & & & & \ddots
\end{array}\right], \quad A_{2}=\left[\begin{array}{cccccc}
\ddots & & & & & \\
\ddots & 0 & & & & \\
\ddots & 1 & \bar{p}_{0} & & & \\
& 0 & q_{0} & 0 & & \\
& & p_{1} & 1 & \bar{p}_{2} & \\
& & 0 & q_{2} & \ddots \\
& & & & p_{3} & \ddots \\
& & & & & \ddots
\end{array}\right],
$$

and $A_{1}$ and $A_{2}$ are invertible; equivalently $\sigma_{2 n-1} \neq 0$ and $q_{2 n} \neq 0$ for all $n$. Due to the symmetry $A=A^{*}$, the generating coefficient sequences are subject to the restrictions (1.9). Note also that degenerations are possible, see Remark 4.3. 
Let $\left\{e_{n}\right\}_{n \in \mathbb{Z}}$ be the standard basis in the two-sided $\ell^{2}$. Thus, constructing $A$ in a generic case, we follow the procedure, which is similar to the CMV matrices case: having $e_{-1}$ and $e_{0}$ as the generators of the cyclic subspace we form the whole space applying $A$ on the even step and $A^{-1}$ on the odd step, however, as it was mentioned, the operator is not unitary, but self-adjoint, that is, the spectrum is not on the unit circle but on the real axis.

Generally, $A$ can be represented as a two dimensional perturbation of a block orthogonal matrix

$$
A=\left[\begin{array}{cc}
A_{-} & 0 \\
0 & A_{+}
\end{array}\right]+e_{-1}\left\langle\cdot, \tilde{e}_{0}\right\rangle \tilde{p}_{0}+\tilde{e}_{0}\left\langle\cdot, e_{-1}\right\rangle \tilde{p}_{0},
$$

where

$$
\tilde{p}_{0}=\left\|P_{+} A e_{-1}\right\|, \quad \tilde{e}_{0}=\frac{1}{\tilde{p}_{0}} P_{+} A e_{-1},
$$

and $A_{ \pm}=P_{ \pm} A P_{ \pm}$are restrictions of $A$ to the positive and negative half-axis according to the orthogonal decomposition $\ell^{2}=\ell_{-}^{2} \oplus \ell_{+}^{2}$. For this reason it is unitary equivalent to an almost periodic finite band Jacobi matrix. In fact $e_{-1}$ and $\tilde{e}_{0}$ are always cyclic for $A$. The corresponding Jacobi matrix $J$ is uniquely defined by the relation

$$
\begin{aligned}
& {\left[\begin{array}{cc}
\left\langle(A-z)^{-1} e_{-1}, e_{-1}\right\rangle & \left\langle(A-z)^{-1} \tilde{e}_{0}, e_{-1}\right\rangle \\
\left\langle(A-z)^{-1} e_{-1}, \tilde{e}_{0}\right\rangle & \left\langle(A-z)^{-1} \tilde{e}_{0}, \tilde{e}_{0}\right\rangle
\end{array}\right]} \\
& \quad=\left[\begin{array}{cc}
\left\langle(J-z)^{-1} e_{-1}, e_{-1}\right\rangle & \left\langle(J-z)^{-1} e_{0}, e_{-1}\right\rangle \\
\left\langle(J-z)^{-1} e_{-1}, e_{0}\right\rangle & \left\langle(J-z)^{-1} e_{0}, e_{0}\right\rangle
\end{array}\right],
\end{aligned}
$$

that is, all general facts from its spectral theory (like the spectral multiplicity) can be reduced to the spectral theory of Jacobi matrices. However it is quite different as soon as we pose the problem:

Problem 1.3. Describe the spectral sets of periodic SMP matrices.

1.3. Main result and an open problem. We consider SMP matrices such that $\|A\|<\infty$ and $\left\|A^{-1}\right\|<\infty$. Therefore in what follows we always assume that the system of intervals $E$ is such that $\infty \notin E$, as well as $0 \notin E$.

Basically from the general theorem of Mumford and van Moerbeke [18] it follows

Theorem 1.4. A set $E$ is a spectrum of a periodic SMP matrix $A$ if and only if the divisor $k\left(\infty_{+}+0_{+}\right)-k\left(\infty_{-}+0_{-}\right)$is a divisor of a function on the Riemann surface $\mathcal{R}$ defined by (1.3), where $k$ is integer, $k \in \mathbb{Z}_{+}$, and $0_{ \pm}$correspond to the origin on the upper and lower sheets of $\mathcal{R}$. Moreover, $2 k$ is a period of $A$. 
To formulate our main result, that is, a description of $E$ in the sense of Marchenko, we define comb regions of a new kind, which we call hyperbolic combs. Recall that the hyperbolic curves

$$
y^{2}-x^{2}=c_{1}, \quad x y=c_{2}
$$

form an orthogonal net in the complex plane $u=x+i y$. The hyperbolas degenerate to pairs of straight lines as $c_{1}=0$ and $c_{2}=0$. The points on such curves will form bases, left/right sides and teeth of the comb.

Definition 1.5. For $h_{0}>0$ the points on the curve

$$
y^{2}-x^{2}=h_{0}^{2}, \quad u=x+i y,
$$

belonging to the upper and lower half-planes, will form the upper and lower bases of a hyperbolic comb $\Pi$, see Figure 1. For integers $k$ and $m \in[0, k]$ and parameter $0 \leq \omega_{0} \leq \pi m$, the upper and lower right sides of $\Pi$ are formed by the points on the curves

$$
2 x y=\pi m-\omega_{0}, \quad 2 x y=\pi(m-k)-\omega_{0}, \quad \text { for } x>0,
$$

and the left ones by

$$
2 x y=-\omega_{0}, \quad 2 x y=\pi k-\omega_{0}, \quad \text { for } x<0 .
$$

The upper and lower teeth of the comb are formed by the points on the curves

$$
2 x y=\pi \ell-\omega_{0}, \quad \text { for } y>0,1 \leq \ell \leq m-1
$$

and

$$
2 x y=\pi(\ell-k)-\omega_{0}, \quad \text { for } y<0, m+1 \leq \ell \leq 2 k-1
$$

starting on the bases and of length $h_{\ell} \geq 0$. In addition, if $\omega_{0}=\pi \ell_{0}$ then the hyperbolic curves related to $\ell=\ell_{0}$ in (1.13) and $\ell=k+\ell_{0}$ in (1.14) degenerate. In this case the corresponding teeth are pieces of the imaginary axis, as soon as

$$
h_{\ell_{0}}<h_{0} \text { and } h_{k+\ell_{0}}<h_{0} \text {. }
$$

Otherwise one of them still satisfies (1.15), and another one has T-shape, see Figure 2, consisting of the piece of the imaginary axis

$$
0 \leq y \leq h_{0} \quad \text { or } \quad-h_{0} \leq y \leq 0
$$

respectively, and of the real interval

$$
-h_{-}^{\left(\ell_{0}\right)} \leq x \leq h_{+}^{\left(\ell_{0}\right)}, \quad h_{ \pm}^{\left(\ell_{0}\right)} \geq 0
$$




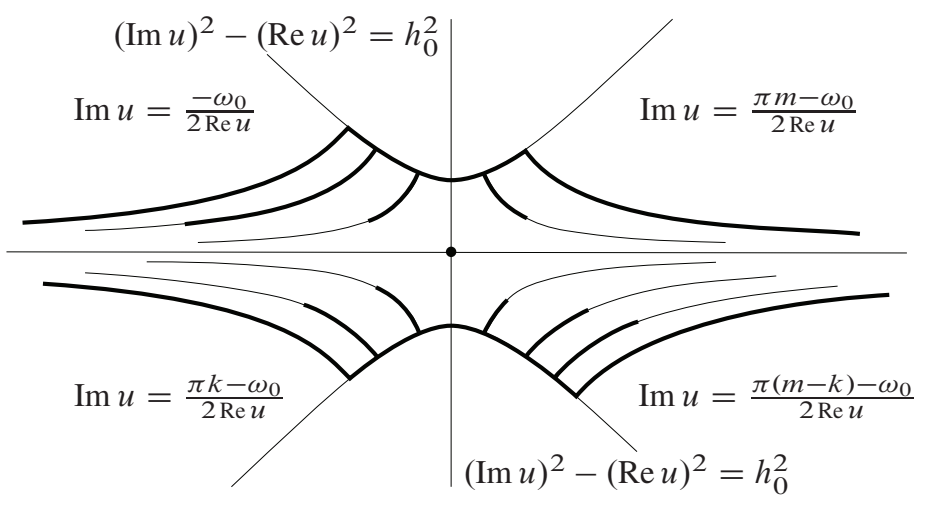

Figure 1. $\Pi$ region for $\omega_{0} \neq \pi \ell$.

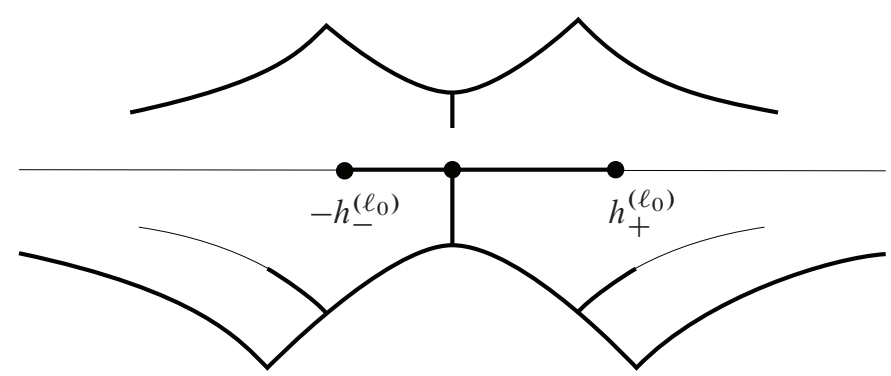

Figure 2. $\Pi$ region for $\omega_{0}=\pi \ell_{0}$.

Remark 1.6. To summarize, a hyperbolic comb $\Pi$ is a domain bounded by hyperbolas with hyperbolic teeth, which depends of the following system of parameters $\Pi=$ $\Pi\left(k, m ; \omega_{0}, h_{0} ; h_{1}, \ldots, h_{2 k-1}\right), k, m \in \mathbb{Z}_{+}, k \geq m, 0 \leq \omega_{0} \leq \pi m, h_{\ell} \geq 0$. Such a domain allows degeneration if $\omega_{0}=\pi \ell_{0}$, case in which two (upper and lower) of the comb's teeth are also given by free independent parameters subject to the trivial restrictions (1.15)-(1.17). Note also that $\Pi$ contains two infinite points corresponding to $x= \pm \infty$. As it was mentioned $2 k$ is a period of $A$ and $m$ describes a "distance" between positions 0 and $\infty$ (in a generic case, how many intervals of $E$ are in between).

Definition 1.7. For a given hyperbolic comb $\Pi$, a comb function $\theta$ is a conformal map $\theta: \mathbb{H} \rightarrow \Pi$ such that 0 and $\infty$ correspond to the infinite points in $\Pi$.

Theorem 1.8. A system of intervals $E$ is the spectral set of a periodic SMP matrix A if and only if it is the preimage through a comb function $\theta$ of the base of a hyperbolic comb $\Pi$. 
Remark 1.9. In Definition 1.7 the conformal mapping $\theta$ is defined up to multiplication by a positive constant $\rho$, i.e., $\theta(\lambda) \mapsto \theta(\rho \lambda)$. This reflects the fact that the transformation $A \mapsto \rho A$ acts on the set of periodic SMP matrices. Similarly, $J \mapsto a J+b, a>0, b \in \mathbb{R}$ acts on the set of periodic Jacobi matrices, and a standard comb defines a spectral set up to the linear transform $\sigma(J) \mapsto a \sigma(J)+b$.

Remark 1.10. Thus, if $E$ is the spectrum of a periodic SMP matrix $A$, then $E$ can be obtain as the preimage of the base for a certain hyperbolic comb $\Pi$. Conversely, if $\Pi$ is given we obtain the system of intervals $E$ as the preimage of the base of this comb (as it was explained in the previous remark, $\Pi$ defines $E$ up to the homogeneous dilation $E \mapsto \rho E$ ). Then, to a such set $E$ one can associate an isospectral set of periodic SMP matrices $A(E)$, which we describe in Theorem 4.2.

In Section 2 we introduce and discuss the spectral surfaces of periodic 5-diagonal matrices of the simplest possible structure. Note that both CMV and SMP matrices are hidden in this class; see (1.18) and (1.19). We do not know how to parametrize (in the Marchenko sense) these spectral surfaces in the general case, see Problem 2.2.

For example, note that the function $z=\lambda+1 / \lambda$ is real on the set $\mathbb{T} \cup \mathbb{R}$. Assume that a measure $\chi$ is supported on a compact subset of $\mathbb{T} \cup \mathbb{R}$ and vanishes in the vicinity of the origin. Then the multiplication by $z$ defines a bounded self-adjoint operator in $L_{\chi}^{2}$. Moreover, with respect to the orthonormal basis (1.6) it is represented by a 5-diagonal (one-sided) matrix, which we denote by $J$ (it is not a Jacobi matrix, since it is indeed 5-diagonal!). Let us point out that if the measure $\chi$ is supported only on $\mathbb{T}$ then

$$
J=\mathfrak{A}+\mathfrak{A}^{-1},
$$

where $\mathfrak{A}$ is a CMV matrix. If $\chi$ is supported only on $\mathbb{R}$ then

$$
J=A+A^{-1},
$$

where $A$ is a SMP matrix. But if the measure does not vanish on both components $\mathbb{T}$ and $\mathbb{R}$ the multiplication by $\lambda$ generates also a certain matrix, which is neither unitary nor self-adjoint, that is, neither of CMV nor of SMP class. We do not know how to describe spectral sets $E$ of corresponding periodic two-sided matrices. Notice that $E$ in this case should be a union of arcs on $\mathbb{T}$ and intervals in $\mathbb{R}$.

The further structure of this work is as follows: in Section 3 we prove our main theorem; the functional model for periodic SMP matrices is given in Section 4.

The second author is thankful to the organizers of the program Hilbert spaces of entire functions and spectral theory of self-adjoint differential operators, at CRM, Barcelona, 2011, and to Alex Eremenko for stimulating discussions. In a sense this paper is an addition to their joint work [11]. 


\section{Spectral surfaces with the maximal number of boundary ovals}

Let $J$ be a 5-diagonal self-adjoint matrix of period $d$

$$
J=r S^{2}+p S+q+S^{-1} \bar{p}+S^{-2} r,
$$

where $S$ is the shift operator and $p, r, q$ are diagonal matrices of period $d$, such that $r_{m}>0$ for all $m \in \mathbb{Z}$ (we say that such $J$ 's are regular). We recall certain fundamental facts from the spectral theory of multi-diagonal periodic matrices [18], adopting to the 5-diagonal case.

For

$$
j(w)=\left[\begin{array}{ccccccc}
q_{0} & \bar{p}_{1} & r_{2} & \ldots & 0 & r_{0} / w & p_{0} / w \\
p_{1} & q_{1} & \bar{p}_{2} & r_{3} & \ldots & 0 & r_{1} / w \\
r_{2} & p_{2} & q_{2} & \bar{p}_{3} & r_{4} & \ldots & 0 \\
\ldots & \ldots & \ldots & \ldots & \ldots & \ldots & \ldots \\
0 & \ldots & r_{d-3} & p_{d-3} & q_{d-3} & \bar{p}_{d-2} & r_{d-1} \\
r_{0} w & 0 & \ldots & r_{d-2} & p_{d-2} & q_{d-2} & \bar{p}_{d-1} \\
\bar{p}_{0} w & r_{1} w & 0 & \ldots & r_{d-1} & p_{d-1} & q_{d-1}
\end{array}\right]
$$

let

$$
F(z, w)=\frac{\operatorname{det}\{j(w)-z \cdot I\}}{\prod_{j=0}^{d-1} r_{j}}=w^{2}+1 / w^{2}+A(z) w+A_{*}(z) 1 / w+B(z)
$$

where $A$ and $B$ are polynomials; in particular, for even $d=2 k$, such that

$$
B(z)=\frac{z^{2 k}}{\prod_{j=0}^{2 k-1} r_{j}}+\cdots,
$$

and

$$
A_{*}(z) \stackrel{\text { def }}{=} \overline{A(\bar{z})}=\left(\frac{-1}{\prod_{j=0}^{k-1} r_{2 j}}+\frac{-1}{\prod_{j=0}^{k-1} r_{2 j+1}}\right) z^{k}+\cdots .
$$

Then the spectral curve corresponding to $J$ is the Riemann surface defined by

$$
\mathcal{R}=\{P=(z, w): F(z, w)=0\}
$$

with an appropriate compactification (adding infinite points). $\mathcal{R}$ is endowed with an antiholomorphic involution $\tau P \stackrel{\text { def }}{=}(\bar{z}, 1 / \bar{w})$ for which

$$
\mathcal{R} \backslash \partial \mathcal{R}_{+}=\mathcal{R}_{+} \cup \mathcal{R}_{-}, \quad \mathcal{R}_{+}=\{P=(z, w) \in \mathcal{R}:|w|<1\} ;
$$

see Figure 3. 


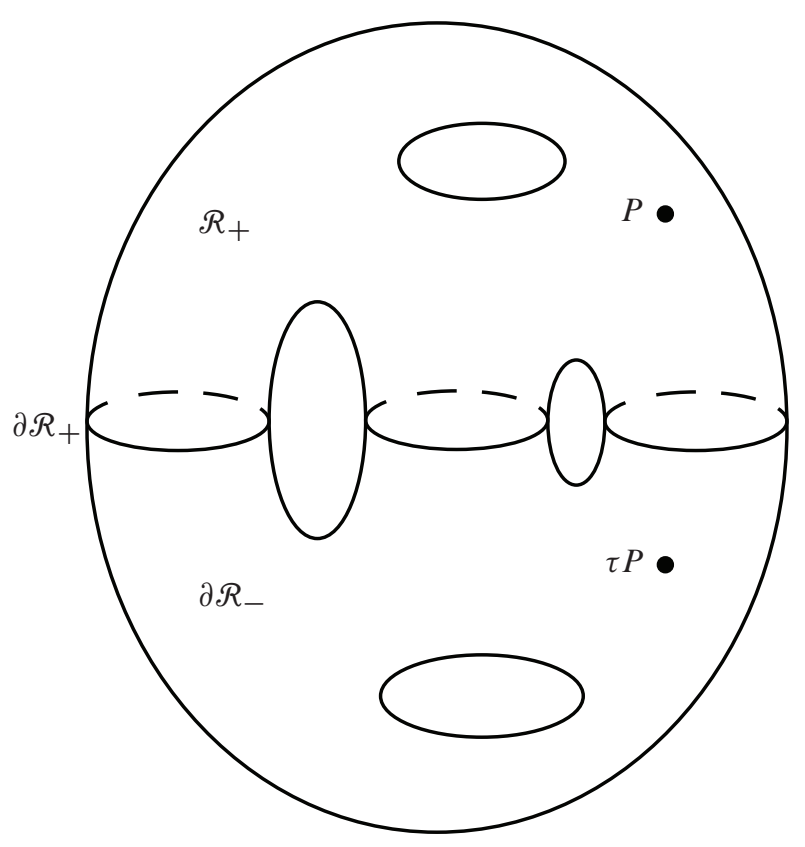

Figure 3. Topology of the spectral curve.

Note that the spectrum of $J$ (as the operator acting in $\ell^{2}$ ) corresponds to the fixed line of the involution $\tau: \tau P=P$,

$z \in \sigma(J)$ if and only if there exists $w$ such that $P=(z, w) \in \partial \mathcal{R}_{+}$.

In other words it is described by the condition $|w|=1$.

Recall that the spectral surfaces related to periodic Jacobi matrices are of the form

$$
\widetilde{\mathcal{R}}=\left\{(w, z): w+\frac{1}{w}=\widetilde{A}(z)\right\},
$$

where $\tilde{A}$ is a real polynomial. In the similar decomposition $\widetilde{\mathcal{R}} \backslash \partial \widetilde{\mathcal{R}}=\widetilde{\mathcal{R}}_{+} \cup \widetilde{\mathcal{R}}_{-}$it possesses the following property: the number of boundary ovals, i.e., the number of intervals

$$
\partial \widetilde{\mathcal{R}}=\{z \in \mathbb{R}:|\widetilde{A}(z)| \leq 2\}
$$

is maximal for the given genus of the surface.

We say that the spectral curve $\mathcal{R}$, related to a 5-diagonal matrix, is of the simplest structure if it has maximal possible number of components of the boundary $\partial \mathcal{R}_{+}$for the given genus. For example, in Figure 3 the number of boundary components is 3 , but its genus is 4 and the maximal possible number of components is 5 . That is, the curve of this structure does not belong to the class. 


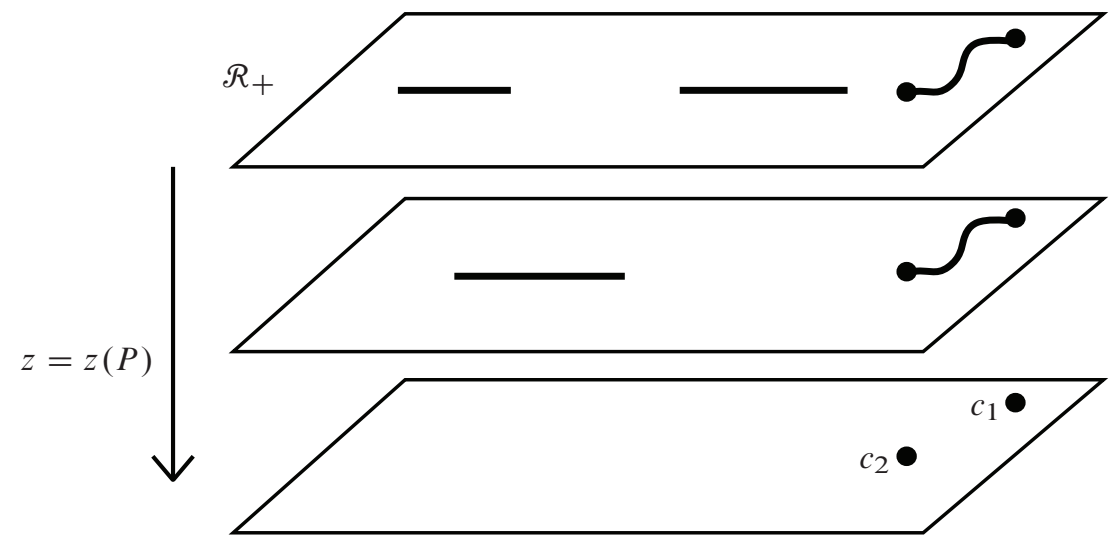

Figure 4. $\mathcal{R}_{+}$as the two sheeted covering of $z$-plane.

In other words, let us represent $\mathcal{R}_{+}$as two a sheeted covering of the $z$-plane, see Figure 4. It is a hyperelliptic curve with a system of cuts $\partial \mathcal{R}_{+}$. We say that the spectral curve is of the simplest structure if this hyperelliptic curve has genus 0, i.e.:

$$
\mathcal{R}_{+} \simeq \overline{\mathbb{C}} \backslash E .
$$

The corresponding equivalence can be written explicitly

$$
z=\lambda+\frac{c^{2}}{\lambda-\lambda_{0}}, \lambda \in \mathbb{C}, \quad c_{1}=\lambda_{0}-2 c, c_{2}=\lambda_{0}+2 c,
$$

where $c_{1}, c_{2}$ denote the only two possible critical values of $z$, in the case that both numbers are finite and if, say, $c_{2}=\infty$ then

$$
z=\lambda^{2}-2 c, \quad c_{1}=c^{2}-2 c .
$$

The set $E$, which corresponds to $\partial \mathcal{R}_{+}$, is a system of cuts in the complex plane $\mathbb{C}$ with the property

$$
z(\lambda) \in \mathbb{R}, \quad \lambda \in E .
$$

It is essential to note that $E$ is far from being an arbitrary system of cuts for which (2.6) holds. Recall that up to now the second function $w$ was not involved into considerations. Meanwhile $w=w(\lambda)$ is a function in $\overline{\mathbb{C}} \backslash E$ with the following properties [18]:

(i) $w$ is single-valued and holomorphic;

(ii) $|w|<1$ in $\overline{\mathbb{C}} \backslash E$ and $|w|=1$ on $E$;

(iii) zeros of $w$ are $\left\{\lambda_{0}, \infty\right\}=z^{-1}(\infty) \not \subset E$ (of equal multiplicity). 
For definiteness, here and below, we consider the case (2.5) (with two finite critical values). Properties (i)-(iii) imply that

$$
\frac{1}{k} \log \frac{1}{|w(\lambda)|}=G_{\lambda_{0}}(\lambda)+G_{\infty}(\lambda)
$$

where $G_{\lambda_{0}}(\lambda)$ is the Green function in the domain $\overline{\mathbb{C}} \backslash E$ with a logarithmic pole at $\lambda_{0}$ and $k$ is the multiplicity of $w$ in $\lambda_{0}$ and $\infty$ respectively.

Let us recall the concept of the complex Green function $b_{\lambda_{0}}(\lambda)$. It is an analytic multivalued function in $\overline{\mathbb{C}} \backslash E$ such that

$$
\log \frac{1}{\left|b_{\lambda_{0}}(\lambda)\right|}=G_{\lambda_{0}}(\lambda)
$$

Note that (2.8) determines $b_{\lambda_{0}}$ up to a unimodular constant. In what follows we assume the normalizations $b_{\lambda_{0}}(\infty)>0$ and $b_{\infty}\left(\lambda_{0}\right)>0$.

Let $\pi_{1}(\overline{\mathbb{C}} \backslash E)$ be the fundamental group of this domain. Then $b_{\lambda_{0}}$ generates the character $\mu_{\lambda_{0}} \in \pi_{1}(\overline{\mathbb{C}} \backslash E)^{*}$ on this group by

$$
b_{\lambda_{0}} \circ \gamma=\mu_{\lambda_{0}}(\gamma) b_{\lambda_{0}}, \quad \gamma \in \pi_{1}(\overline{\mathbb{C}} \backslash E),
$$

which indicates the multivalued structure of the complex Green function. Moreover, let us split $E$ into connected components, $E=\bigcup_{j=0}^{\alpha} E_{j}$, and let $\gamma_{j}$ 's be simple contours around $E_{j}$ 's. Note that they form generators of the group $\pi_{1}(\overline{\mathbb{C}} \backslash E)$ subject to the condition

$$
\gamma_{0} \circ \cdots \circ \gamma_{m}=\text { trivial. }
$$

Then, for a suitable choice of the direction of $\gamma_{j}$,

$$
\mu_{\lambda_{0}}\left(\gamma_{j}\right)=e^{2 \pi i \omega_{\lambda_{0}}\left(E_{j}\right)},
$$

where $\omega_{\lambda_{0}}\left(E_{j}\right)$ is the harmonic measure of $E_{j}$ at $\lambda_{0}$.

The factor $b_{\lambda_{0}} b_{\infty}$ removes the singularities of $z$ in $\overline{\mathbb{C}} \backslash E$. Let

$$
t_{\infty}=\frac{\left(b_{\infty} b_{\lambda_{0}} z\right)(\infty)}{\left|\left(b_{\infty} b_{\lambda_{0}} z\right)(\infty)\right|} \quad \text { and } \quad t_{\lambda_{0}}=\frac{\left(b_{\infty} b_{\lambda_{0}} z\right)\left(\lambda_{0}\right)}{\left|\left(b_{\infty} b_{\lambda_{0}} z\right)\left(\lambda_{0}\right)\right|} .
$$

Define $\xi \in[0,1)$ by the condition $t_{\infty}=e^{2 \pi i \xi} t_{\lambda_{0}}$. Let $\mu=\mu_{\lambda_{0}} \mu_{\infty}$. Thus $\mu\left(\gamma_{j}\right)=$ $e^{2 \pi i \omega_{j}}$, where $\omega_{j}=\omega_{\lambda_{0}}\left(E_{j}\right)+\omega_{\infty}\left(E_{j}\right)$.

Theorem 2.1. Let $z$ be given by (2.5). Let $E=\bigcup_{j=0}^{\varkappa} E_{j} \subset z^{-1}(\mathbb{R})$ be a system of cuts (2.6). Then $\mathcal{R}=\mathcal{R}_{+} \cup \partial \mathcal{R}_{+} \cup \mathcal{R}_{-}$, where $\mathcal{R}_{+} \simeq \overline{\mathbb{C}} \backslash E$, is a spectral curve of a 5-diagonal periodic matrix if and only if the numbers $\xi$ and $\omega_{j}$ (for all $j$ ) are rational. Moreover $w=t_{\infty}^{-k}\left(b_{\lambda_{0}} b_{\infty}\right)^{k}$, where $k$ is a common denominator of these rational numbers. 
Problem 2.2. Find a parametric description of sets $E$ from Theorem 2.1 in the Marchenko sense.

A proof of Theorem 2.1 is based on a fact of the general theory [18], that a given periodic $J$ with the spectral curve $\mathcal{R}$ possesses functional representation as the multiplication operator by $z$. We give such a representation following basically [27] and [32].

For a fixed character $\alpha$ the multivalued analytic functions $F, F \circ \gamma=\alpha(\gamma) F$, such that $|F(\lambda)|^{2}$ has a harmonic majorant in $\overline{\mathbb{C}} \backslash E$, form the Hardy space $H^{2}(\alpha) \subset L_{\omega_{\infty}}^{2}$ with the norm given by the integral of the boundary values:

$$
\|F\|^{2}=\int_{E}|F(\lambda)|^{2} \omega_{\infty}(d \lambda) .
$$

Note that the point-evaluation functional is bounded in this space and therefore in $H^{2}(\alpha)$ there is the reproducing kernel $k_{\lambda}^{\alpha}$ :

$$
F(\lambda)=\left\langle F, k_{\lambda}^{\alpha}\right\rangle, \quad \lambda \in \overline{\mathbb{C}} \backslash E,
$$

for all $F \in H^{2}(\alpha)$.

Lemma 2.3. For a character $\alpha \in \pi_{1}(\overline{\mathbb{C}} \backslash E)^{*}$ let

$$
K_{\lambda}^{\alpha}=\frac{k_{\lambda}^{\alpha}}{\left\|k_{\lambda}^{\alpha}\right\|}
$$

denote the normalized reproducing kernel at $\lambda$. Then, for an arbitrary system of unimodular constants $t_{m}$, the family

$$
e_{2 n}=t_{2 n} b_{\lambda_{0}}^{n} b_{\infty}^{n} K_{\lambda_{0}}^{\alpha \mu \lambda_{0}^{-n} \mu_{\infty}^{-n}}, \quad e_{2 n+1}=t_{2 n+1} b_{\lambda_{0}}^{n+1} b_{\infty}^{n} K_{\infty}^{\alpha \mu_{\lambda_{0}}^{-n-1} \mu_{\infty}^{-n}}
$$

forms an orthonormal basis in $H^{2}(\alpha), n \geq 0$. Moreover, extended to negative indexes it forms an orthonormal basis in $L_{\omega_{\infty}}^{2}$.

Proof. The system (2.10) is orthonormal: indeed, for $m \leq n$,

$$
\begin{aligned}
\left\langle e_{2 n}, e_{2 m-1}\right\rangle & =\left\langle t_{2 n} b_{\lambda_{0}}^{n} b_{\infty}^{n} K_{\lambda_{0}}^{\alpha \mu_{\lambda_{0}}^{-n} \mu_{\infty}^{-n}}, t_{2 m-1} b_{\lambda_{0}}^{m} b_{\infty}^{m-1} K_{\infty}^{\alpha \mu} \lambda_{\lambda_{0}}^{-m} \mu_{\infty}^{-m+1}\right\rangle \\
& =\left\langle t_{2 n} b_{\lambda_{0}}^{n-m} b_{\infty}^{n-m+1} K_{\lambda_{0}}^{\alpha \mu_{\lambda_{0}}^{-n} \mu_{\infty}^{-n}}, t_{2 m-1} K_{\infty}^{\left.\alpha \mu_{\lambda_{0}}^{-m} \mu_{\infty}^{-m+1}\right\rangle}\right\rangle \\
& =\overline{t_{2 m-1}} t_{2 n} b_{\lambda_{0}}^{n-m}(\infty) b_{\infty}^{n-m+1}(\infty) \frac{K_{\lambda_{0}}^{\alpha \mu_{\lambda_{0}}^{-n} \mu_{\infty}^{-n}}(\infty)}{K_{\infty}^{\alpha \mu_{\lambda_{0}}^{-m} \mu_{\infty}^{-m+1}}(\infty)}=0,
\end{aligned}
$$


where in the last line we used the fact that $K_{\infty}^{\alpha \mu_{\lambda_{0}}^{-m}} \mu_{\infty}^{-m+1}$ is the normalized reproducing kernel and $b_{\infty}(\infty)=0$. All other possible relations between the indexes of the basis elements can be treated in a similar way.

Moreover, every function from $H^{2}(\alpha)$ orthogonal to it has a zero of infinite multiplicity in $\lambda_{0}$ (and $\infty$ ) and therefore vanishes identically. To prove the second claim one has to use the description of the orthogonal complement $L_{\omega_{\infty}}^{2} \ominus H^{2}(\alpha)$ by means of the Hardy space [32] and once again apply the same argument related to the corresponding $\mathrm{H}^{2}$-space and orthonormal basis of reproducing kernels in it.

Lemma 2.4. The multiplication by $z$ with respect to the basis (2.10) is a 5-diagonal self-adjoint matrix,

$$
z e_{m+2}=\bar{r}_{m+2} e_{m}+\bar{p}_{m+2} e_{m+1}+q_{m+2} e_{m+2}+p_{m+3} e_{m+3}+r_{m+4} e_{m+4} .
$$

Moreover $r_{m}>0$ if and only if

$$
t_{2 n-1}=t_{\infty}^{-n} t_{(-1)}, \quad t_{2 n}=t_{\lambda_{0}}^{-n} t_{(0)} .
$$

Proof. Since the factor $b_{\lambda_{0}} b_{\infty}$ removes the singularities of $z$ in $\overline{\mathbb{C}} \backslash E$ the function $z b_{\lambda_{0}} b_{\infty} F$ belongs to $H^{2}(\alpha \mu)$ for every $F \in H^{2}(\alpha)$ and an arbitrary $\alpha \in \pi_{1}(\overline{\mathbb{C}} \backslash E)^{*}$. Thus the decomposition of $z e_{m+2}$ starts with $e_{m}$,

$$
z e_{m+2}=\bar{r}_{m+2} e_{m}+\cdots \text {. }
$$

Since $z(\lambda)$ is real on $E$ the multiplication operator is self-adjoint; its matrix possesses the symmetry property and therefore it is 5-diagonal (2.11). Finally we put $\lambda=\lambda_{0}$ in (2.11) for even $m$

$$
\left(z b_{\lambda_{0}} b_{\infty}\right)\left(\lambda_{0}\right) K_{\lambda_{0}}^{\alpha \mu^{-(n+2)}}\left(\lambda_{0}\right) t_{2 n+2}=\bar{r}_{2 n+2} K_{\lambda_{0}}^{\alpha \mu^{-n}}\left(\lambda_{0}\right) t_{2 n}
$$

and $\lambda=\infty$ for odd $m$

$$
\left(z b_{\lambda_{0}} b_{\infty}\right)(\infty) K_{\infty}^{\alpha \mu_{\infty} \mu^{-(n+2)}}(\infty) t_{2 n+1}=\bar{r}_{2 n+1} K_{\infty}^{\alpha \mu_{\infty} \mu^{-n}}(\infty) t_{2 n-1} .
$$

Since $K_{\lambda}^{\alpha}(\lambda)>0$ we get (2.12).

Proof of Theorem 2.1. Let $J$ be a periodic self-adjoint 5-diagonal matrix and $\mathcal{R}$ be its spectral surface such that $\mathcal{R}_{+} \simeq \overline{\mathbb{C}} \backslash E$. Since $w(\lambda)$ is single-valued in the domain, (2.7) and (2.9) imply that $\omega_{j}=\omega_{\lambda_{0}}\left(E_{j}\right)+\omega_{\infty}\left(E_{j}\right)$ are rational. Further, due to (2.3) the function $w z^{k}$ is regular in the domain, moreover $1 /\left(w z^{k}\right)\left(\lambda_{0}\right)$ and $1 /\left(w z^{k}\right)(\infty)$ are roots of the quadratic equation

$$
\begin{aligned}
C^{2} & +\left(\frac{-1}{\prod_{j=0}^{k-1} r_{2 j}}+\frac{-1}{\prod_{j=0}^{k-1} r_{2 j+1}}\right) C+\frac{1}{\prod_{j=0}^{2 k-1} r_{j}} \\
& =\left(C-\frac{1}{\prod_{j=0}^{k-1} r_{2 j}}\right)\left(C-\frac{1}{\prod_{j=0}^{k-1} r_{2 j}}\right) \\
& =0 .
\end{aligned}
$$


Thus $\left(w z^{k}\right)\left(\lambda_{0}\right)>0$ and $\left(w z^{k}\right)(\infty)>0$. Since $w=t\left(b_{\lambda_{0}} b_{\infty}\right)^{k}, t \in \mathbb{T}$, the ratio $\left(z b_{\lambda_{0}} b_{\infty}\right)^{k}(\infty) /\left(z b_{\lambda_{0}} b_{\infty}\right)^{k}\left(\lambda_{0}\right)$ is also positive. That is $e^{2 \pi i k \xi}=1$. And this finishes the necessity part of the theorem.

In the opposite direction, for the given system of cuts we define $J$ according to Lemma 2.4. It remains to check that $J$ is periodic. Let $w=t_{\infty}^{-k}\left(b_{\lambda_{0}} b_{\infty}\right)^{k}$. Since $\mu^{k}(\gamma)=1$, for all $\gamma \in \pi_{1}(\overline{\mathbb{C}} \backslash E)$, it is single-valued. Note that $w$ is normalized by the condition $\left(w z^{k}\right)(\infty)=\left|\left(b_{\lambda_{0}} b_{\infty} z\right)^{k}(\infty)\right|>0$. We claim that

$$
w e_{n}=e_{n+2 k} \text {. }
$$

For odd $n$, equation (2.13) holds automatically. For even $n$ we should take into account that in addition $t_{\infty}^{-k} t_{\lambda_{0}}^{k}=e^{-2 \pi i k \xi}=1$. Thus (2.13) defines the shift operator. Since the multiplication operators by $z$ and $w$ commute, we have $J S^{2 k}=S^{2 k} J$. Therefore $J$ is periodic.

Now, let us restrict ourselves to the real case, i.e., $c_{2}=\bar{c}_{1}$ or both critical values are real. Without lost of generality $c_{2}=\bar{c}_{1}=2 i$ or $c_{2}=-c_{1}=2$. Thus, according to $(2.5)$,

$$
z=\lambda-\frac{1}{\lambda}
$$

in the first case, and

$$
z=\lambda+\frac{1}{\lambda}
$$

in the second one.

In the case $(2.14), z^{-1}(\mathbb{R})=\mathbb{R}$, thus $E$ is a system of intervals on the real axis. Since $z^{-1}(\infty)=\{0, \infty\}, E$ is subject to the restriction $\{0, \infty\} \not \subset E$.

If $z$ is of the form $(2.15)$, then $z^{-1}(\mathbb{R})=\mathbb{R} \cup \mathbb{T}$, that is, $E$ is a union of real intervals and arcs of the unit circle, and again $\{0, \infty\} \not \subset E$. This case under the additional assumption $E \subset \mathbb{T}$ leads to periodic CMV matrices [30]. Indeed, in the current case the multiplication by $\lambda$ is also well defined and represents an unitary matrix $A$ such that

$$
J=A+A^{-1}=A+A^{*} .
$$

As it was mentioned this functional model is the same as that related to periodic and almost periodic CMV matrices, see e.g. [27]. Conversely, having a periodic CMV matrix $A$ we obtain the periodic self-adjoint $J$ of the class (2.16).

Similarly, in the case (2.14) the multiplication by $\lambda$ leads to the self-adjoint operator $A$ such that

$$
J=A-A^{-1}
$$

where $A^{-1}$ exists and corresponds to the multiplication by $1 / \lambda$, i.e., to a periodic SMP matrix. Further details of the corresponding functional model are discussed in Section 4. Note that the case (2.15) under the additional assumption $E \subset \mathbb{R}$ leads to essentially the same class of self-adjoint operators.

Thus our formal definition for SMP matrices is as follows. 
Definition 2.5. A self-adjoint 5-diagonal matrix $A$ of the form

$$
A e_{m+2}=r_{m+2} e_{m}+\bar{p}_{m+2} e_{m+1}+q_{m+2} e_{m+2}+p_{m+3} e_{m+3}+r_{m+4} e_{m+4},
$$

where $r_{2 n}=0$ and $r_{2 n+1}>0$, belongs to the SMP class if $A$ is invertible and $-S^{-1} A^{-1} S$ belongs to the same class.

Remark 2.6. We say that $A$ of the form (2.18) belongs to the $S M P_{+}$class if the involution in the class is given by $A \mapsto S^{-1} A^{-1} S$.

\section{Proof of the Main Theorem}

Let $E=\left[b_{0}, a_{0}\right] \backslash \bigcup_{j=1}^{\varkappa}\left(a_{j}, b_{j}\right)$ be a system of intervals on $\mathbb{R}$, recall $\{0, \infty\} \in \overline{\mathbb{C}} \backslash E$, $z=\lambda-1 / \lambda$. We apply Theorem 2.1 in the current case. As it is well known the Green function (say with respect to infinity) is represented by the hyperelliptic integral; see e.g. [33], and [32],

$$
G(\lambda, \infty)=\operatorname{Re} \int_{a_{0}}^{\lambda} \frac{\lambda^{\varkappa}+\cdots}{\sqrt{\prod_{j=0}^{\varkappa}\left(\lambda-a_{j}\right)\left(\lambda-b_{j}\right)}} d \lambda .
$$

Therefore for the sum of the Green functions we have

$$
G(\lambda, \infty)+G(\lambda, 0)=\operatorname{Re} \int_{a_{0}}^{\lambda} \frac{M_{\varkappa+1}(\lambda)}{\sqrt{\prod_{j=0}^{\varkappa}\left(\lambda-a_{j}\right)\left(\lambda-b_{j}\right)}} \frac{d \lambda}{\lambda},
$$

where $M_{\varkappa+1}$ is a monic polynomial of degree $\varkappa+1$. Note that the residue of the corresponding differential at the origin is -1 .

3.1. Spectrum of SMP matrices for the Stieltjes class. Let us consider the simplest cases $E \subset \mathbb{R}_{+}$or $E \subset \mathbb{R}_{-}$(we can say that the spectrum is on the upper (lower) sheet of $\left.\mathcal{R}_{+}\right)$. The strong Stieltjes moment problem is related to measures supported on the positive half-axis [19]. The shape of the sum $G(\lambda, \infty)+G(\lambda, 0)$ on $\mathbb{R} \backslash E$ is shown in Figure 5. It implies immediately that all the zeros of the polynomial $M_{\varkappa+1}$ in (3.1) are real in this case. Indeed, each gap $\left(a_{j}, b_{j}\right), j \geq 1$, contains at least one critical point; there is a critical point between $-\infty$ and 0 ; the total number of critical points is $\varkappa+1$. Therefore

$$
\tilde{\theta}(\lambda)=i \int_{a_{0}}^{\lambda} \frac{M_{\varkappa+1}(\lambda)}{\sqrt{\prod_{j=0}^{\varkappa}\left(\lambda-a_{j}\right)\left(\lambda-b_{j}\right)}} \frac{d \lambda}{\lambda}
$$

is the Schwarz-Christoffel integral, which maps conformally the upper half-plane $\mathbb{\square}$ onto the (generalized) polygon in Figure 6. 


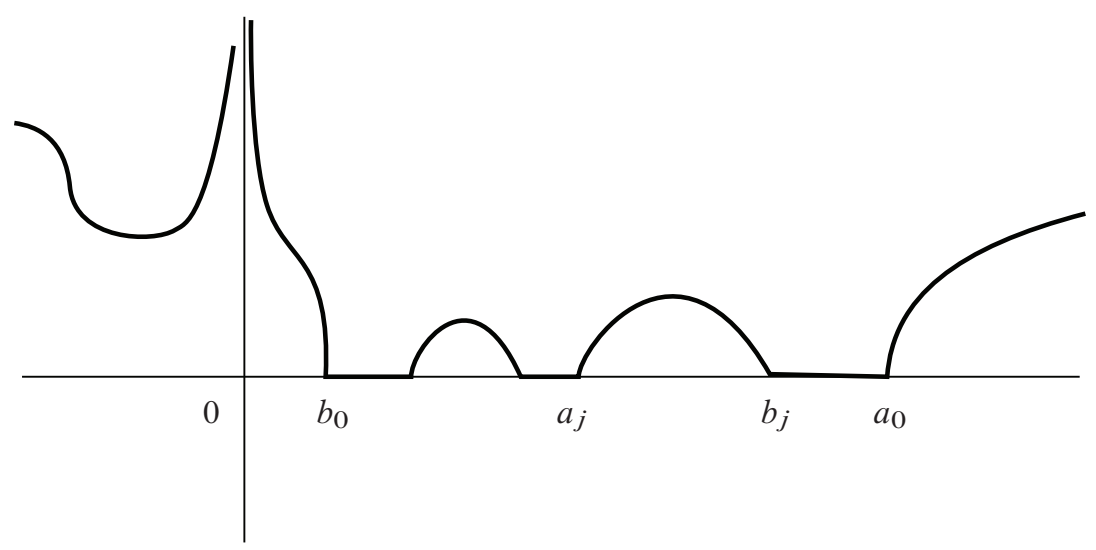

Figure 5. $G_{0}+G_{\infty}$ on $\mathbb{R}$ : the spectrum is on the upper sheet.

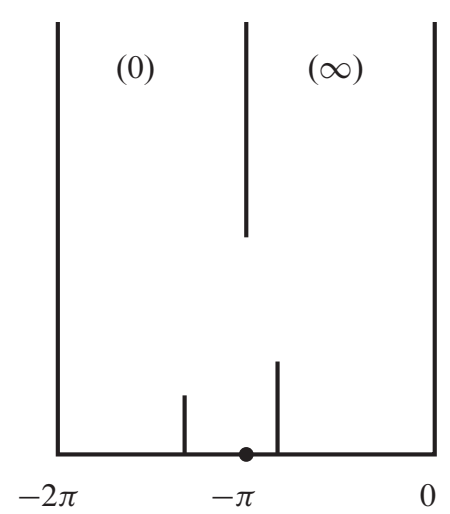

Figure 6. Image of the Abelian integral $\tilde{\theta}$.

According to (2.7) $w(\lambda)=t e^{i k \tilde{\theta}(\lambda)}, t \in \mathbb{T}$. Let $\tilde{\omega}_{j}$ be the coordinates of the base of the slits. Then $w(\lambda)$ is single valued in $\overline{\mathbb{C}} \backslash E$ if and only if $\tilde{\omega}_{j} k \in \pi \mathbb{Z}$ for all $j$. It remains to mention that due to the chosen normalization for $b_{\lambda_{0}}$ and $b_{\infty}$ the product $b_{\lambda_{0}}(\lambda) b_{\infty}(\lambda)(\lambda-1 / \lambda)$ is positive at infinity and negative at the origin, that is $\xi=1 / 2$. Thus we can parametrize the spectral sets of periodic SMP matrices in this case by sufficiently simple domains shown in Figure 6 with rational $\tilde{\omega}_{j}$ 's (quite similar to the Jacobi and CMV matrices cases).

3.2. Complex critical points and three real critical points in the same gap. The situation changes dramatically as soon as $0 \in\left(a_{j}, b_{j}\right), j \geq 1$, that is, $E=E_{-} \cup E_{+}$, $E_{ \pm} \subset \mathbb{R}_{ \pm}$. Still all gaps, except for $\left(a_{j}, b_{j}\right)$, should contain a critical point, thus $M_{\varkappa+1}$ has at least $\varkappa-1$ real critical points. However, the positions of two remaining critical points are not a priory fixed. 
First, we consider the case when two remaining critical points are complex $\mu_{0}$ and $\overline{\mu_{0}}, \operatorname{Im} \mu_{0}>0$. Let us consider $\tilde{\theta}(\lambda)$ in the upper half-plane $\mathbb{H}$. Since locally $\tilde{\theta}(\lambda)=\tilde{\theta}\left(\mu_{0}\right)+C\left(\lambda-\mu_{0}\right)^{2}+\cdots$, there exist two orthogonal directions where $\operatorname{Re} d \tilde{\theta}=0$. Moreover for one of them $\operatorname{Im} \tilde{\theta}$ has a local minimum at $\mu_{0}$ and a local maximum for another one. We define the curve $\gamma, \mu_{0} \in \gamma$, by the condition $\operatorname{Re} d \tilde{\theta}=0$, such that $\operatorname{Im} \tilde{\theta}$ increases. Since there is no other critical point in $\mathbb{H}$ this curve should terminate on the real axis. Note that $\operatorname{Im} \tilde{\theta}(\lambda)$ decreases as $\lambda$ approaches $E$. If so, in the gaps $\gamma$ may approach either a critical point or 0 and $\infty$. The first case is also not possible since the critical point is a local minimum for $\operatorname{Im} \tilde{\theta}=G_{\lambda_{0}}+G_{\infty}$ along the real axis, thus it should be local maximum in the orthogonal direction $\gamma$. But along $\gamma$ it increases. Thus, $\gamma$ terminates at 0 and $\infty$; see Figure 7.

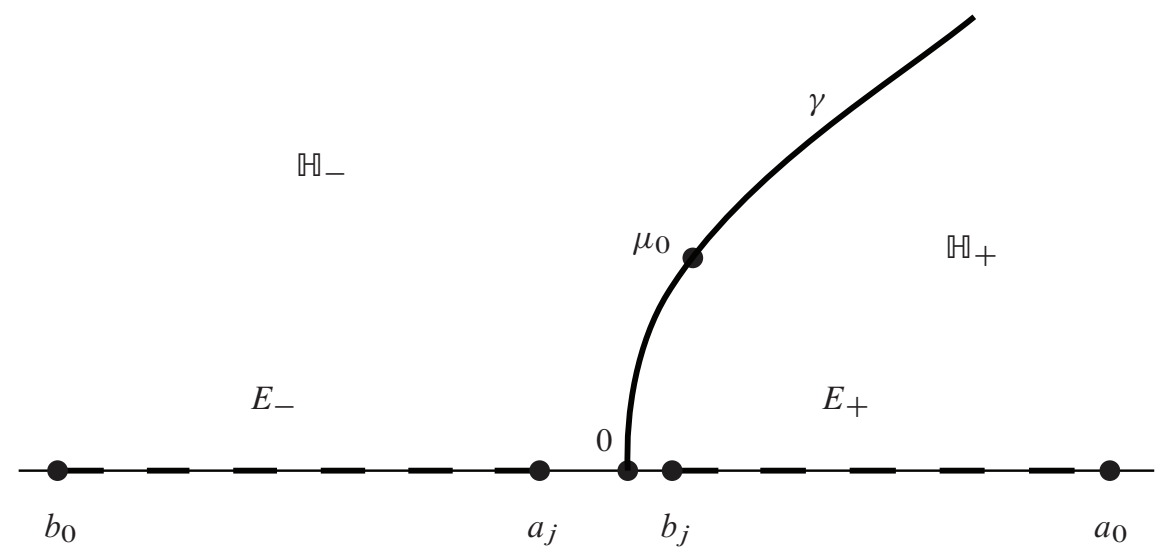

Figure 7. A complex critical point.

As the result we get $\mathbb{H} \backslash \gamma=\mathbb{H}_{-} \cup \mathbb{H}_{+}$such that $E_{ \pm} \subset \partial \mathbb{H}_{ \pm}$. Let $\theta(\lambda)=k \tilde{\theta}(\lambda)$. Inspecting the boundary behavior of the given analytic function we obtain that it maps conformally $\mathbb{H}_{ \pm}$onto $D_{ \pm}$shown in Figure 8 , where the point $-\omega_{0}+i h_{0}^{2}$ corresponds to the critical point $\mu_{0}$.

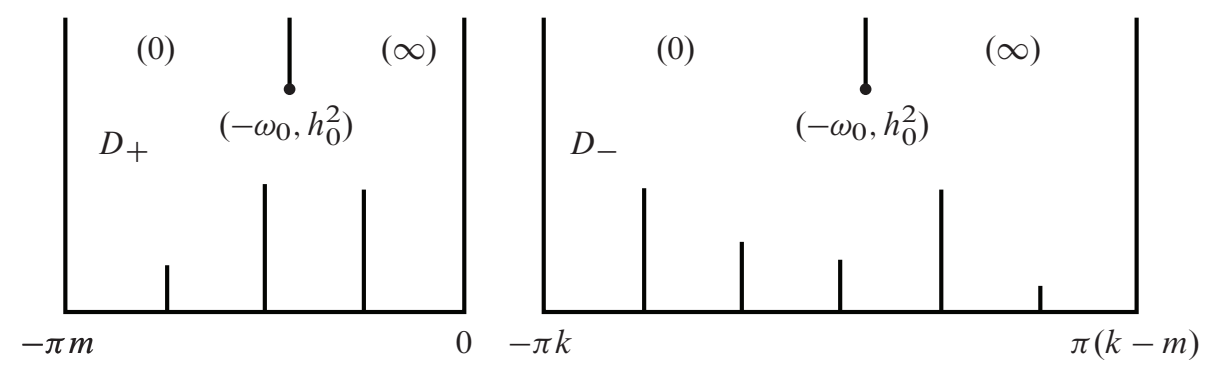

Figure 8. $D_{ \pm}=\theta\left(\mathbb{H}_{ \pm}\right)$regions for a complex critical point. 
Now we define

$$
u(\lambda)=\sqrt{-i \theta(\lambda)-i \omega_{0}-h_{0}^{2}}, \quad \lambda \in \mathbb{H}_{+} .
$$

Here we assume that $\operatorname{Im} u(\lambda)>0$. Similarly we define

$$
u(\lambda)=-\sqrt{-i \theta(\lambda)-i \omega_{0}-h_{0}^{2}}, \quad \lambda \in \mathbb{R}_{-}
$$

and in this case $\operatorname{Im} u(\lambda)<0$. In this way we get the regions $\Pi_{ \pm}$. Since

$$
\theta=-\left(2 \operatorname{Re} u \operatorname{Im} u+\omega_{0}\right)+i\left((\operatorname{Re} u)^{2}-(\operatorname{Im} u)^{2}+h_{0}^{2}\right),
$$

these regions are bounded by hyperbolic curves (1.10)-(1.14); see Figure 1. Gluing the images along the curve $\gamma$ we obtain the conformal mapping of the upper half-plane $\mathbb{W}$ onto the special comb domain $\Pi=\Pi_{+} \cup \Pi_{-} \cup \mathbb{R}$.

Conversely, for the region $\Pi$ described by these equations we define a conformal map $u: \mathbb{U} \rightarrow \Pi, u(0)=+\infty, u(\infty)=-\infty$, and set

$$
w(\lambda)=e^{-\left(u^{2}(\lambda)+h_{0}^{2}+i \omega_{0}\right)}, \quad z=\lambda-\frac{1}{\lambda} .
$$

Then, the set $E$ corresponds to $|w|=1$. Since the base of the slits for $D_{ \pm}$are of the form $\pi \ell, w$ extended in the lower half-plane is single-valued in $\overline{\mathbb{C}} \backslash E$. Finally, $w$ is real for $\lambda \in \mathbb{R} \backslash E$, that is, $\xi$ is rational. Based on Theorem 2.1 we conclude that this domain can be associated to a periodic SMP matrix.

Let us turn to the case of three real critical points in the same gap. In this case W can be decomposed into three pieces. Let $\mu_{1}<\mu_{0}<\mu_{2}$ be critical points in the gap $\left(a_{i}, b_{i}\right)$. Note that with necessity $\mu_{1}$ and $\mu_{2}$ are points of local maximum and $\operatorname{Im} \theta$ assumes a local minimum at $\mu_{0}$ in this interval. Therefore there are directions $\gamma_{1}, \gamma_{2}$ orthogonal to the real axis at $\mu_{1}$ and $\mu_{2}$ respectively such that $\operatorname{Im} \theta$ increases. Arguments like the above show that these curves, $\operatorname{Re} d \theta=0$, terminate at 0 and $\infty$; see Figure 9.

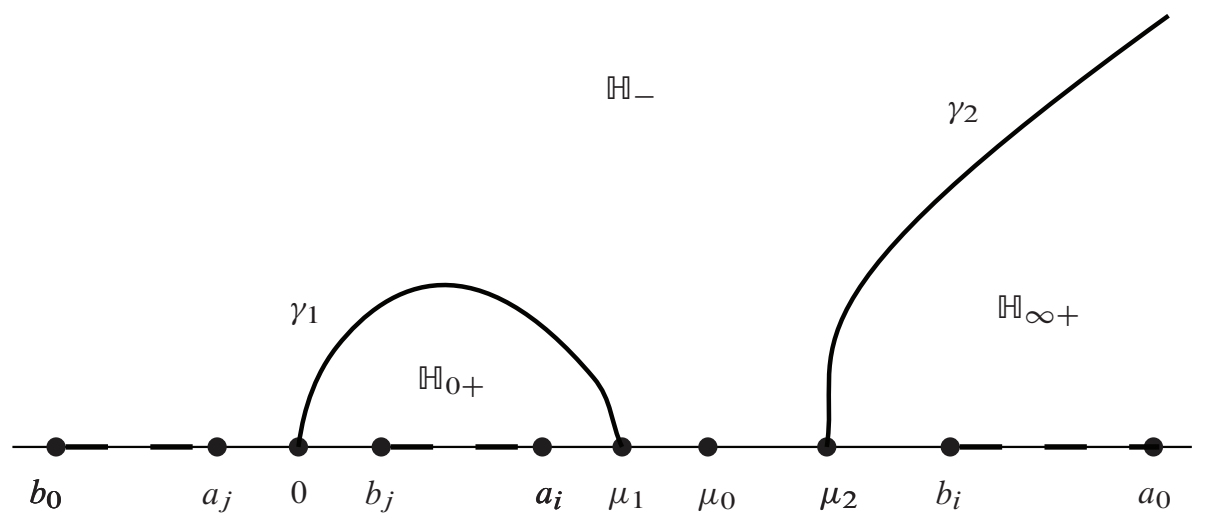

Figure 9. Three real critical points in the same gap, $\mathbb{}_{+}=\mathbb{\sharp}_{0+} \cup \mathbb{\sharp}_{\infty+}$. 
In each of them, $\theta(\lambda)$ represents a conformal mapping, see Figure 10. In this picture $-\omega_{0}+i h_{0}^{2},-\omega_{0}+i h_{1}^{2}$, and $-\omega_{0}+i h_{2}^{2}$ are images of the critical points $\mu_{0}, \mu_{1}$, and $\mu_{2}$ respectively and $\omega_{0}=\pi \ell_{0}$. We make the change of variable (3.3) and (3.4), having in mind that now $\mathbb{H}_{+}$or $\mathbb{H}_{-}$consists of two components. We arrive at the parametrization of the spectral curve by the domains of the form Figure 2 such that

$$
h_{-}^{\left(\ell_{0}\right)}=\sqrt{h_{2}^{2}-h_{0}^{2}} \text { and } h_{+}^{\left(\ell_{0}\right)}=\sqrt{h_{1}^{2}-h_{0}^{2}}
$$

in (1.17).

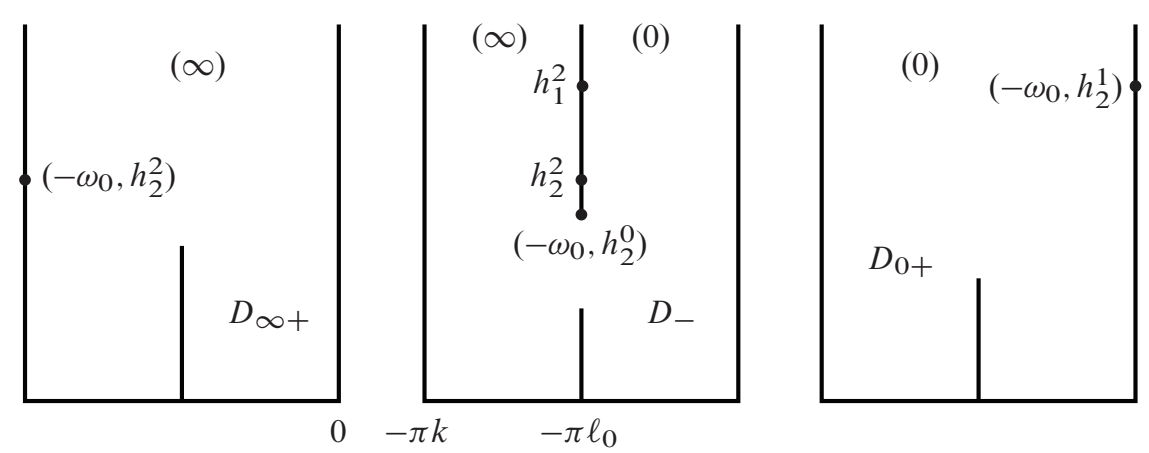

Figure 10. $D_{ \pm}=\theta\left(\mathbb{H}_{ \pm}\right)$three real critical points in the same gap.

As before, starting from a region $\Pi$, by (3.5) we arrive at the set $E$ and the domain $\overline{\mathbb{C}} \backslash E \simeq \mathcal{R}_{+}$which corresponds to a periodic SMP matrix.

3.3. Other cases. In the previous subsection we considered critical values in two main generic positions. Now let us list the remaining special cases.

1. For two complex critical values, if $\omega_{0}=\pi \ell_{0}$ then one of the cuts in (1.13) and one in (1.14) degenerate to the intervals on the imaginary axis. The length of such a cut can not be arbitrary long, thus $h_{\ell_{0}}$ and $h_{k+\ell_{0}}$ are subject for conditions (1.15). As soon as one of these values approaches $h_{0}$ two complex critical values, from the upper and lower half-planes, approach the critical value in the corresponding gap. In the limit we have the critical value of multiplicity 3 . The same special case can be obtained when two critical values $\mu_{1}$ and $\mu_{2}$ tend to $\mu_{0}$, correspondingly $h_{ \pm}^{\left(\ell_{0}\right)} \rightarrow 0$; see Figure 2.

2. The case of a critical point of multiplicity two and a simple critical point in a gap corresponds to $h_{+}^{\left(\ell_{0}\right)}=0, h_{-}^{\left(\ell_{0}\right)}>0$ or $h_{-}^{\left(\ell_{0}\right)}=0, h_{+}^{\left(\ell_{0}\right)}>0$.

3. Two critical points (or one critical point of multiplicity two) may appear in the interval which contains zero or infinity. The domain $\Pi$ looks similar to that one shown in Figure 2, but the degenerated hyperbola corresponds to the most left (or right) position, i.e., $\ell_{0}=0$ or $\ell_{0}=m$. 
4. It was assumed that $m \leq k$. If $m>k$ the domain $\Pi$ in Figure 1 remains the same, but we switch the normalization conditions to $u(0)=-\infty$ and $u(\infty)=+\infty$.

5. In the Stieltjes case, subsection 3.1, the spectral curve was described by a simpler domain, Figure 6. By (3.3) it can be transformed to a $\Pi$ region bounded from below by the real axis.

\section{Functional model for periodic SMP matrices}

Let $\overline{\mathbb{C}} \backslash E$ correspond to a periodic SMP matrix. We define

$$
\mathcal{A}(\alpha)=\frac{K_{0}^{\alpha}(\infty)}{K_{\infty}^{\alpha}(\infty)}, \quad \mathcal{B}(\alpha)=\frac{K_{0}^{\alpha}(0)}{K_{\infty}^{\alpha}(\infty)}, \quad \alpha \in \pi_{1}(\overline{\mathbb{C}} \backslash E)^{*} .
$$

For the reader's convenience we prove here a known lemma, see e.g. [27].

Lemma 4.1. The following identities hold true

$$
\mathcal{C}(\alpha) \stackrel{\text { def }}{=} \sqrt{1-|\mathcal{A}(\alpha)|^{2}}=b_{\infty}(0) \frac{K_{0}^{\alpha \mu_{\infty}^{-1}}(0)}{K_{0}^{\alpha}(0)}
$$

and

$$
\overline{\mathcal{A}(\alpha)}=\frac{K_{\infty}^{\alpha}(0)}{K_{0}^{\alpha}(0)}, \quad e(\alpha)=b_{0}(\infty) \frac{K_{\infty}^{\alpha \mu_{0}^{-1}}(\infty)}{K_{\infty}^{\alpha}(\infty)} .
$$

Proof. $\mathcal{A}(\alpha)$ is defined by the following orthogonal decompositions:

$$
\begin{aligned}
K_{0}^{\alpha} & =\mathcal{A}(\alpha) K_{\infty}^{\alpha} \quad+\sqrt{1-|\mathcal{A}(\alpha)|^{2}} b_{\infty} K_{0}^{\alpha \mu_{\infty}^{-1}} \\
b_{0} K_{\infty}^{\alpha \mu_{0}^{-1}} & =\sqrt{1-|\mathcal{A}(\alpha)|^{2}} K_{\infty}^{\alpha}-\overline{\mathcal{A}(\alpha)} b_{\infty} K_{0}^{\alpha \mu_{\infty}^{-1}}
\end{aligned}
$$

Indeed,

$$
\mathcal{A}(\alpha)=\frac{K_{0}^{\alpha}(\infty)}{K_{\infty}^{\alpha}(\infty)}
$$

and we get (4.2).

Since, in addition,

$$
\begin{aligned}
K_{\infty}^{\alpha} & =\overline{\mathcal{A}(\alpha)} K_{0}^{\alpha}+\sqrt{1-|\mathcal{A}(\alpha)|^{2}} b_{0} K_{\infty}^{\alpha \mu_{0}^{-1}} \\
b_{\infty} K_{0}^{\alpha \mu_{\infty}^{-1}} & =\sqrt{1-|\mathcal{A}(\alpha)|^{2}} K_{0}^{\alpha}-\quad \mathcal{A}(\alpha) b_{0} K_{\infty}^{\alpha \mu_{0}^{-1}}
\end{aligned}
$$

we have (4.3). 
In what follows without loss of generality we assume that $t_{(-1)}=1$ in (2.12). In the given case $\lambda_{0}=0$, so $t_{0}$ is the new notation for $t_{\lambda_{0}}$, and this is not the same as the initial $t_{(0)}$. Since

$$
\left(b_{\infty} b_{0} z\right)(\infty)=\left(b_{\infty} \lambda\right)(\infty) b_{0}(\infty) \quad \text { and } \quad\left(b_{\infty} b_{0} z\right)(0)=-\left(b_{0} / \lambda\right)(0) b_{\infty}(0),
$$

we have $t_{\infty}=\varphi_{\infty} /\left|\varphi_{\infty}\right|$ and $t_{0}=-\varphi_{0} /\left|\varphi_{0}\right|$, where

$$
\varphi_{\infty}=\left(\frac{b_{\infty} \lambda}{b_{0}}\right)(\infty) \text { and } \varphi_{0}=\left(\frac{b_{0}}{b_{\infty} \lambda}\right)(0) .
$$

Also, recall that $t_{\infty} / t_{0}=e^{2 \pi i \xi}$.

Theorem 4.2. The multiplication operator by $\lambda$ with respect to the basis (2.10) is a periodic SMP matrix $A=A\left(\alpha, t_{(0)}\right)$ with the following coefficients

$$
\begin{aligned}
& \bar{p}_{2 n}=\varphi_{\infty} t_{(0)} e^{2 \pi i \xi n} \mathcal{A}\left(\alpha \mu^{-n}\right) \mathcal{B}^{-1}\left(\alpha \mu^{-n}\right) \bigodot\left(\alpha \mu_{\infty} \mu^{-n}\right) \mathscr{B}\left(\alpha \mu_{\infty} \mu^{-n}\right), \\
& p_{2 n+1}=-\bar{\varphi}_{\infty} t_{(0)} t_{\infty} e^{2 \pi i \xi n} \bigodot\left(\alpha \mu^{-n}\right) \mathcal{B}\left(\alpha \mu^{-n}\right) \mathcal{A}\left(\alpha \mu_{\infty} \mu^{-n}\right) \mathcal{B}^{-1}\left(\alpha \mu_{\infty} \mu^{-n}\right), \\
& q_{2 n}=-\varphi_{\infty} \mathcal{A}\left(\alpha \mu^{-n}\right) \mathcal{B}^{-1}\left(\alpha \mu^{-n}\right) \overline{\mathcal{A}\left(\alpha \mu_{\infty} \mu^{-n}\right)} \mathscr{B}\left(\alpha \mu_{\infty} \mu^{-n}\right),
\end{aligned}
$$

and

$$
\begin{gathered}
\pi_{2 n+1}=t_{(0)} t_{\infty} e^{2 \pi i \xi n} \bar{\varphi}_{0} \varphi\left(\alpha \mu^{-n}\right) \mathcal{B}\left(\alpha \mu^{-n}\right) \mathcal{A}\left(\alpha \mu_{\infty} \mu^{-(n+1)}\right) \mathcal{B}^{-1}\left(\alpha \mu_{\infty} \mu^{-(n+1)}\right), \\
\bar{\pi}_{2 n+2}=-t_{(0)} e^{2 \pi i \xi(n+1)} \varphi_{0} \mathcal{A}\left(\alpha \mu^{-n}\right) \mathcal{B}^{-1}\left(\alpha \mu^{-n}\right) \\
\qquad\left(\alpha \mu_{\infty} \mu^{-(n+1)}\right) \mathscr{B}\left(\alpha \mu_{\infty} \mu^{-(n+1)}\right), \\
\sigma_{2 n+1}=-\varphi_{0} \mathcal{A}\left(\alpha \mu^{-n}\right) \mathcal{B}^{-1}\left(\alpha \mu^{-n}\right) \overline{\mathcal{A}\left(\alpha \mu_{\infty} \mu^{-(n+1)}\right)} \mathscr{B}\left(\alpha \mu_{\infty} \mu^{-(n+1)}\right) .
\end{gathered}
$$

Proof. Note that $b_{\infty} \lambda k_{0}^{\alpha} \in H^{2}\left(\alpha \mu_{\infty}\right)$ and it is orthogonal to $b_{0} b_{\infty}^{2} H^{2}\left(\alpha \mu_{0}^{-1} \mu_{\infty}^{-2}\right)$. Therefore, in fact, we have the three-terms recurrence relation,

$$
\lambda e_{2 n}=\bar{p}_{2 n} e_{2 n-1}+q_{2 n} e_{2 n}+p_{2 n+1} e_{2 n+1} .
$$

Moreover

$$
\bar{p}_{2 n}=t_{(0)} e^{2 \pi i \xi n}\left(\lambda b_{\infty}\right)(\infty) \frac{K_{0}^{\alpha \mu^{-n}}(\infty)}{K_{\infty}^{\alpha \mu_{\infty} \mu^{-n}}(\infty)}
$$




$$
\begin{aligned}
q_{2 n} & =\left\langle\lambda K_{0}^{\alpha \mu^{-n}}, K_{0}^{\alpha \mu^{-n}}\right\rangle \\
& =\left\langle\lambda K_{0}^{\alpha \mu^{-n}}-\frac{\left(\lambda b_{\infty}\right)(\infty) K_{\infty}^{\alpha \mu_{\infty} \mu^{-n}}}{b_{\infty} K_{\infty}^{\alpha \mu_{\infty} \mu^{-n}}(\infty)} K_{0}^{\alpha \mu^{-n}}(\infty), K_{0}^{\alpha \mu^{-n}}\right\rangle \\
& =-\frac{\left(\lambda b_{\infty}\right)(\infty)}{b_{\infty}(0)} \frac{K_{\infty}^{\alpha \mu_{\infty} \mu^{-n}}(0)}{K_{\infty}^{\alpha \mu_{\infty} \mu^{-n}}(\infty)} \frac{K_{0}^{\alpha \mu^{-n}}(\infty)}{K_{0}^{\alpha \mu^{-n}}(0)},
\end{aligned}
$$

and

$$
\begin{aligned}
& p_{2 n+1} \\
& =\left\langle\lambda t_{(0)} t_{0}^{-n} K_{0}^{\alpha \mu^{-n}}, t_{\infty}^{-n-1} b_{0} K_{\infty}^{\alpha \mu_{0}^{-1} \mu^{-n}}\right\rangle \\
& =t_{(0)} e^{2 \pi i \xi n} t_{\infty}\left\langle K_{0}^{\alpha \mu^{-n}},\right. \\
& \left.\quad \lambda b_{0} K_{\infty}^{\alpha \mu_{0}^{-1} \mu^{-n}}-\frac{\left(\lambda b_{0} b_{\infty}\right)(\infty) K_{\infty}^{\alpha \mu_{0}^{-1} \mu^{-n}}(\infty) K_{\infty}^{\alpha \mu_{\infty} \mu^{-n}}}{b_{\infty} K_{\infty}^{\alpha \mu} \mu^{-n}(\infty)}\right\rangle \\
& \quad \frac{\left(\lambda b_{\infty}\right)(\infty)}{b_{\infty}(0)} \frac{K_{\infty}^{\alpha \mu_{\infty} \mu^{-n}}(0)}{K_{\infty}^{\alpha \mu_{\infty} \mu^{-n}}(\infty)} \frac{b_{0}(\infty) K_{\infty}^{\alpha \mu_{0}^{-1} \mu^{-n}}(\infty)}{K_{0}^{\alpha \mu^{-n}}(0)} .
\end{aligned}
$$

In its turn,

$$
\frac{1}{\lambda} e_{2 n+1}=\bar{\pi}_{2 n+1} e_{2 n}+\sigma_{2 n+1} e_{2 n+1}+\pi_{2 n+2} e_{2 n+2},
$$

where

$$
\begin{gathered}
\bar{\pi}_{2 n+1}=\bar{t}_{(0)} \bar{t}_{\infty} e^{-2 \pi i \xi n}\left(\frac{b_{0}}{\lambda}\right)(0) \frac{K_{\infty}^{\alpha \mu_{0}^{-1} \mu^{-n}}(0)}{K_{0}^{\alpha \mu^{-n}}(0)}, \\
\sigma_{2 n+1}=\left\langle\frac{1}{\lambda} K_{\infty}^{\alpha \mu_{0}^{-1} \mu^{-n}}, K_{\infty}^{\alpha \mu_{0}^{-1} \mu^{-n}}\right\rangle \\
=\left\langle\frac{1}{\lambda} K_{\infty}^{\alpha \mu_{0}^{-1} \mu^{-n}}-\left(\frac{b_{0}}{\lambda}\right)(0) \frac{K_{0}^{\alpha \mu^{-n}}}{b_{0} K_{0}^{\alpha \mu^{-n}}(0)} K_{\infty}^{\alpha \mu_{0}^{-1} \mu^{-n}}(0), K_{\infty}^{\alpha \mu_{0}^{-1} \mu^{-n}}\right\rangle \\
=-\left(\frac{b_{0}}{\lambda}\right)(0) \frac{1}{b_{0}(\infty)} \frac{K_{0}^{\alpha \mu^{-n}}(\infty)}{K_{0}^{\alpha \mu^{-n}}(0)} \frac{K_{\infty}^{\alpha \mu_{0}^{-1} \mu^{-n}}(0)}{K_{\infty}^{\alpha \mu_{0}^{-1} \mu^{-n}}(\infty)},
\end{gathered}
$$


and

$$
\begin{aligned}
& \pi_{2 n+2} \\
& =\left\langle\frac{1}{\lambda} t_{\infty}^{-n-1} K_{\infty}^{\alpha \mu_{0}^{-1} \mu^{-n}}, t_{(0)} t_{0}^{-n-1} b_{\infty} K_{0}^{\alpha \mu^{-1} \mu^{-n}}\right\rangle \\
& =\bar{t}_{(0)} e^{-2 \pi i \xi(n+1)}\left\langle K_{\infty}^{\alpha \mu_{0}^{-1} \mu^{-n}},\right. \\
& \left.\frac{b_{\infty}}{\lambda} K_{0}^{\alpha \mu^{-n-1}}-\frac{K_{0}^{\alpha \mu^{-n}}\left(\frac{b_{0} b_{\infty}}{\lambda} K_{0}^{\alpha \mu^{-n-1}}\right)(0)}{b_{0} K_{0}^{\alpha \mu^{-n}}(0)}\right\rangle \\
& =-\bar{t}_{(0)} e^{-2 \pi i \xi(n+1)}\left(\frac{b_{0} b_{\infty}}{\lambda}\right)(0) \frac{1}{b_{0}(\infty)} \frac{K_{0}^{\alpha \mu^{-n}}(\infty)}{K_{0}^{\alpha \mu^{-n}}(0)} \frac{K_{0}^{\alpha \mu^{-n-1}}(0)}{K_{\infty}^{\alpha \mu_{0}^{-1} \mu^{-n}}(\infty)}
\end{aligned}
$$

Now by making use of (4.1), (4.3), and (4.2), we obtain (4.4)

$$
\begin{aligned}
& q_{2 n}=-\varphi_{\infty} \overline{\mathcal{A}\left(\alpha \mu_{\infty} \mu^{-n}\right)} \mathcal{B}\left(\alpha \mu_{\infty} \mu^{-n}\right) \mathcal{A}\left(\alpha \mu^{-n}\right) \mathcal{B}^{-1}\left(\alpha \mu^{-n}\right), \\
& \bar{p}_{2 n}=\varphi_{\infty} t_{(0)} e^{2 \pi i \xi n} b_{\infty}(0) \frac{K_{0}^{\alpha \mu^{-n}}(\infty)}{K_{\infty}^{\alpha \mu \mu^{-n}}(\infty)} \\
& =\varphi_{\infty} t_{(0)} e^{2 \pi i \xi n} \frac{K_{0}^{\alpha \mu^{-n}}(\infty)}{K_{\infty}^{\alpha \mu^{-n}}(\infty)} \frac{K_{\infty}^{\alpha \mu^{-n}}(\infty)}{K_{\infty}^{\alpha \mu_{\infty} \mu^{-n}}(\infty)} b_{\infty}(0) \\
& =\varphi_{\infty} t_{(0)} e^{2 \pi i \xi n} \mathcal{A}\left(\alpha \mu^{-n}\right) \mathcal{B}^{-1}\left(\alpha \mu^{-n}\right) \mathcal{C}\left(\alpha \mu_{\infty} \mu^{-n}\right) \mathscr{B}\left(\alpha \mu_{\infty} \mu^{-n}\right) \text {, } \\
& \bar{p}_{2 n+1}=-\varphi_{\infty} \bar{t}_{(0)} \bar{t}_{\infty} e^{-2 \pi i \xi n} \overline{\mathcal{A}\left(\alpha \mu_{\infty} \mu^{-n}\right)} \mathcal{B}\left(\alpha \mu_{\infty} \mu^{-n}\right) \\
& \times \frac{b_{0}(\infty) K_{\infty}^{\alpha \mu_{0}^{-1} \mu^{-n}}(\infty)}{K_{\infty}^{\alpha \mu^{-n}}(\infty)} \frac{K_{\infty}^{\alpha \mu^{-n}}(\infty)}{K_{0}^{\alpha \mu^{-n}}(0)} \\
& =-\varphi_{\infty} \bar{t}_{(0)} \bar{t}_{\infty} e^{-2 \pi i \xi n} \overline{\mathcal{A}\left(\alpha \mu_{\infty} \mu^{-n}\right)} \mathscr{B}\left(\alpha \mu_{\infty} \mu^{-n}\right) \mathcal{C}\left(\alpha \mu^{-n}\right) \mathscr{B}^{-1}\left(\alpha \mu^{-n}\right) \text {, }
\end{aligned}
$$

as well as (4.5)

$$
\begin{aligned}
& \bar{\pi}_{2 n+1} \\
& =\bar{t}_{(0)} \bar{t}_{\infty} e^{-2 \pi i \xi n} \varphi_{0} \frac{K_{\infty}^{\alpha \mu_{0}^{-1} \mu^{-n}}(0)}{K_{\infty}^{\alpha \mu_{0}^{-1} \mu^{-n}}(\infty)} b_{0}(\infty) \frac{K_{\infty}^{\alpha \mu_{0}^{-1} \mu^{-n}}(\infty)}{K_{\infty}^{\alpha \mu^{-n}}(\infty)} \frac{K_{\infty}^{\alpha \mu^{-n}}(\infty)}{K_{0}^{\alpha \mu^{-n}}(0)} \\
& =\bar{t}_{(0)} \bar{t}_{\infty} e^{-2 \pi i \xi n} \varphi_{0} \overline{\mathcal{A}\left(\alpha \mu_{\infty} \mu^{-(1+n)}\right)} \mathcal{B}\left(\alpha \mu_{\infty} \mu^{-(1+n)}\right) \mathcal{C}\left(\alpha \mu^{-n}\right) \mathcal{B}^{-1}\left(\alpha \mu^{-n}\right), \\
& \sigma_{2 n+1}=-\varphi_{0} \mathcal{A}\left(\alpha \mu^{-n}\right) \mathcal{B}^{-1}\left(\alpha \mu^{-n}\right) \overline{\mathcal{A}\left(\alpha \mu_{\infty} \mu^{-(n+1)}\right)} \mathscr{B}\left(\alpha \mu_{\infty} \mu^{-(n+1)}\right),
\end{aligned}
$$


and

$$
\begin{aligned}
\bar{\pi}_{2 n+2}=-t_{(0)} e^{2 \pi i \xi(n+1)} \varphi_{0} \mathcal{A}\left(\alpha \mu^{-n}\right) \mathcal{B}^{-1}\left(\alpha \mu^{-n}\right) \\
\quad \times b_{0}(\infty) \frac{K_{0}^{\alpha \mu^{-(n+1)}}(0)}{K_{0}^{\alpha \mu_{0}^{-(n+1)}}(0)} \frac{K_{0}^{\alpha \mu_{0}^{-1} \mu^{-n}}(0)}{K_{\infty}^{\alpha \mu_{0}^{-1} \mu^{-n}}(\infty)} \\
=-t_{(0)} e^{2 \pi i \xi(n+1)} \varphi_{0} \mathcal{A}\left(\alpha \mu^{-n}\right) \mathcal{B}^{-1}\left(\alpha \mu^{-n}\right) \\
e\left(\alpha \mu_{\infty} \mu^{-(n+1)}\right) \mathscr{B}\left(\alpha \mu_{\infty} \mu^{-(n+1)}\right) .
\end{aligned}
$$

Remark 4.3. The structure of the reproducing kernels on the hyperelliptic Riemann surfaces is well known, see e.g. [32]. In particular, indeed $K_{\infty}^{\alpha}(0)=0$, i.e., for some $\alpha, \mathcal{A}(\alpha)=0$. According to (4.4) and (4.5) it means that the corresponding $A=$ $A\left(\alpha, t_{(0)}\right)$ may degenerate, that is, $q_{2 n}$ or $\sigma_{2 n-1}$ vanishes for some $n$. Nevertheless all entries of $A$ and $A^{-1}$ have perfect sense. For example,

$$
\begin{aligned}
r_{2 n+1} & =\frac{\bar{p}_{2 n} \bar{p}_{2 n+1}}{q_{2 n}} \\
& =\left|\varphi_{\infty}\right| \bigodot\left(\alpha \mu^{-n}\right) \mathscr{B}\left(\alpha \mu^{-n}\right) \circlearrowright\left(\alpha \mu_{\infty} \mu^{-n}\right) \mathcal{B}^{-1}\left(\alpha \mu_{\infty} \mu^{-n}\right),
\end{aligned}
$$

and

$$
\begin{aligned}
-\rho_{2 n}= & \frac{\bar{\pi}_{2 n-1} \bar{\pi}_{2 n}}{-\sigma_{2 n-1}} \\
& =\left|\varphi_{0}\right| \bigodot\left(\alpha \mu^{-(n-1)}\right) \mathscr{B}\left(\alpha \mu^{-(n-1)}\right) \bigodot\left(\alpha \mu_{\infty} \mu^{-n}\right) B^{-1}\left(\alpha \mu_{\infty} \mu^{-n}\right),
\end{aligned}
$$

where

$$
A e_{2 n-1}=r_{2 n-1} e_{2 n-3}+\bar{p}_{2 n-1} e_{2 n-2}+q_{2 n-1} e_{2 n-1}+p_{2 n} e_{2 n}+r_{2 n+1} e_{2 n+1},
$$

and

$$
A^{-1} e_{2 n}=\rho_{2 n} e_{2 n-2}+\bar{\pi}_{2 n} e_{2 n-1}+\sigma_{2 n} e_{2 n}+\pi_{2 n+1} e_{2 n+1}+\rho_{2 n+2} e_{2 n+2} .
$$

\section{References}

[1] N. Akhiezer and B. Levin, Generalization of S. N. Bernstein's inequality for derivatives of entire functions. In Issledovanija po sovremennym problemam teorii funkcii kompleksnogo peremennogo. Gosudarstv. Izdat. Fiz.-Mat. Lit., Moscow, 1960, 111-165. In Russian. MR 0125968

[2] A. I. Aptekarev, Asymptotic properties of polynomials orthogonal on a system of contours, and periodic motions of Toda chains. Mat. Sb. (N.S.) 125 (1984), 231-258. In Russian. MR 0764479

[3] A. Bogatyrev, A combinatorial description of a moduli space of curves and of extremal polynomials. Mat. Sb. 194 (2003), 27-48. English Transl. Sb. Math. 194 (2003), 1451-1473. MR 2037514 Zbl 1097.14021 
[4] A. Bogatyrev, Extremal polynomials and Riemann surfaces. Moskovskii Tsentr Nepreryvnogo Matematicheskogo Obrazovaniya, Moscow, 2005. English transl. Springer Monographs in Mathematics. Springer, Berlin etc., 2012. MR 2287691 MR 2953752 (transl.) Zbl 1243.65001 Zbl 1252.30001 (transl.)

[5] J. Breuer, E. Ryckman, and M. Zinchenko, Right limits and reflectionless measures for CMV matrices. Comm. Math. Phys. 292 (2009), 1-28. MR 2540069 Zbl 1188.35172

[6] A. Bultheel, P. González-Vera, E. Hendriksen, and O. Njåstad, Natural solutions of rational Stieltjes moment problems. J. Math. Anal. Appl. 377 (2011), 571-583. MR 2769158 Zbl 1211.44006

[7] S. Clark and F. Gesztesy, Weyl-Titchmarsh theory for singular finite difference Hamiltonian systems. J. Comput. Appl. Math. 171 (2004), 151-184.MR 2077203Zbl 1065.39027

[8] S. Clark, F. Gesztesy, and W. Renger, Trace formulas and Borg-type theorems for matrixvalued Jacobi and Dirac finite difference operators. J. Differential Equations 219 (2005), 144-182. MR 2181033 Zbl 1085.39016

[9] D. Damanik, R. Killip, and B. Simon, Perturbations of orthogonal polynomials with periodic recursion coefficients. Ann. of Math. (2) 171 (2010), 1931-2010. MR 2680401 Zbl 1194.47031

[10] K. Deckers and D.S. Lubinsky, How poles of orthogonal rational functions affect their Christoffel functions. J. Approx. Theory 164 (2012), 1184-1999. MR 2948560 Zbl 1254.42033

[11] A. Eremenko and P. Yuditskii, Comb functions. In J. Arvesú and G. López Lagomasino (eds.), Recent advances in orthogonal polynomials, special functions, and their applications. Proceedings of the $11^{\text {th }}$ International Symposium (OPSFA'11) held at the Universidad Carlos III de Madrid, Leganés, August 29-September 2, 2011. Contemporary Mathematics 578. American Mathematical Society, Providence, RI, 2012, 99-118. MR 2964141 Zbl 1250.00015 (collection)

[12] F. Gesztesy, A. Kiselev, and K. Makarov, Uniqueness results for matrix-valued Schrödinger, Jacobi, and Dirac-type operators. Math. Nachr. 239/240 (2002), 103-145. MR 1905666 Zbl 1017.34020

[13] S. Khrushchev, The Euler-Lagrange theory for Schur's algorithm: algebraic exposed points. J. Approx. Theory 139 (2006), 402-429. MR 2220047 Zbl 1090.11007

[14] S. Khrushchev, Periodic Schur functions and slit discs. J. Approx. Theory 159 (2009), 293-307. MR 2562746 Zbl 1204.30002

[15] R. Killip and I. Nenciu, CMV: the unitary analogue of Jacobi matrices. Comm. Pure Appl. Math. 60 (2007), 1148-1188. MR 2330626 Zbl 1128.15012

[16] E. Korotyaev and A. Kutsenko, Lyapunov functions of periodic matrix-valued Jacobi operators. In T. Suslina and D. Yafaev (eds.), Spectral theory of differential operators. M. Sh. Birman $80^{\text {th }}$ anniversary collection. American Mathematical Society Translations, Series 2, 225. Advances in the Mathematical Sciences 62. American Mathematical Society, Providence, RI, 2008, 117-131. MR 2509779 Zbl 1173.47019

[17] V. Marchenko and I. Ostrovskii, A characterization of the spectrum of the Hill operator. Mat. Sb. (N.S.) 97 (1975), 540-606 and 633-634. English transl. Math. USSRSb. 26(1975) (1977), 493-554. MR 0409965 Zbl 0343.34016 
[18] P. van Moerbekke and D. Mumford, The spectrum of difference operators and algebraic curves. Acta Math. 143 (1979), 93-154. MR 0533894 Zbl 0502.58032

[19] O. Njåstad, Solutions of the strong Hamburger moment problem. J. Math. Anal. Appl. 197 (1996), 227-248. MR 1371286 Zbl 0853.44004

[20] F. Peherstorfer, On Bernstein-Szegö orthogonal polynomials on several intervals. II. Orthogonal polynomials with periodic recurrence coefficients. J. Approx. Theory 64 (1991), 123-161. MR 1091466 Zbl 0721.42017

[21] F. Peherstorfer, Orthogonal and extremal polynomials on several intervals. J. Comput. Appl. Math. 48 (1993), 187-205. MR 1246858 Zbl 0790.42012

[22] F. Peherstorfer, Minimal polynomials on several intervals with respect to the maximum norm. A survey. In A. Martínez Finkelshtein, F. Marcellán and J. J. Moreno-Balcázar (eds.), Complex methods in approximation theory. Proceedings of the Workshop on Methods of Complex Analysis in Approximation Theory held at the University of Almería, Almería, June 5-8, 1995. Monografías Ciencia y Tecnología 2. Universidad de Almería, Servicio de Publicaciones, Almería, 1997, 137-159. MR 1635621 Zbl 0904.41018

[23] F. Peherstorfer and R. Steinbauer, Orthogonal polynomials on arcs of the unit circle I. J. Approx. Theory 85 (1996), 140-184. MR 1385813 Zbl 0861.42014

[24] F. Peherstorfer and R. Steinbauer, Orthogonal polynomials on arcs of the unit circle. II. Orthogonal polynomials with periodic reflection coefficients. J. Approx. Theory 87 (1996), 60-102. MR 1410612 Zbl 0946.42016

[25] F. Peherstorfer, Orthogonal and extremal polynomials on several intervals. In J. S. Dehesa (ed.) Proceedings of the Seventh Spanish Symposium on Orthogonal Polynomials and Applications (VII SPOA). Held at the University of Granada, Granada, September 23-27, 1991. J. Comput. Appl. Math. 48 (1993), Elsevier Science, Amsterdam, 1993, 187-205. MR 1246858 Zbl 0790.42012

[26] F. Peherstorfer and P. Yuditskii, Almost periodic Verblunsky coefficients and reproducing kernels on Riemann surfaces. J. Approx. Theory 139 (2006), 91-106. MR 2220035 Zbl 1103.30007

[27] F. Peherstorfer, and P. Yuditskii, Finite difference operators with a finite band spectrum. In J. Janas, P. Kurasov, A. Laptev, S. Naboko, and G. Stolz, Methods of spectral analysis in mathematical physics. Proceedings of the International Conference on Operator Theory, Analysis and Mathematical Physics (OTAMP 2006) held in Lund, June 2006. Operator Theory: Advances and Applications 186. Birkhäuser Verlag, Basel, 2009, 347-389. MR 2732087 Zbl 1169.47020

[28] L. V. Perkolab, An inverse problem for a periodic Jacobi matrix. Teor. Funktsii Funktsional. Anal. i Prilozhen. 42 (1984), 107-121. In Russian. MR 0751397

[29] B. Simon, Orthogonal polynomials on the unit circle. I. Classical theory. American Mathematical Society Colloquium Publications 54, Part 1. American Mathematical Society, Providence, RI, 2005. MR 2105088 Zbl 1082.42020

[30] B. Simon, Orthogonal polynomials on the unit circle. II. Spectral theory. American Mathematical Society Colloquium Publications 54, Part 2. American Mathematical Society, Providence, RI, 2005. MR 2105089 Zbl 1082.42021 
[31] M. Sodin and P. Yuditskii, Functions that deviate least from zero on closed subsets of the real axis. Algebra i Analiz 4 (1992), 1-61. English transl. St. Petersburg Math. J. 4 (1993), 201-249. MR 1182392

[32] M. Sodin and P. Yuditskii, Almost periodic Jacobi matrices with homogeneous spectrum, infinite dimensional Jacobi inversion, and Hardy spaces of character-automorphic functions. J. Geom. Anal. 7 (1997), 387-435. MR 1674798 Zbl 1041.47502

[33] Yu. Tomchuk, Orthogonal polynomials over a system of intervals on the real line. Zap. Fiz.-Mat. Fak. i Kharkov. Mat. Obshch. 29 (1963), 93-128.

Received February 13, 2012; revised October 24, 2012

Ionela Moale, Research Institute for Symbolic Computation,

Johannes Kepler University Linz, Altenbergerstr. 69, A-4040, Linz, Austria

E-mail: Ionela.Moale@ risc.jku.at

Peter Yuditskii, Abteilung für Dynamische Systeme und Approximationstheorie,

Johannes Kepler Universität Linz, A-4040 Linz, Austria

E-mail: Petro.Yudytskiy@jku.at 